\title{
Exact controllability of fractional neutral integro-differential systems with state-dependent delay in Banach spaces
}

\author{
S. Kailasavalli, D. Baleanu, S. Suganya and M. Mallika Arjunan
}

\begin{abstract}
In this manuscript, we have a tendency to execute Banach contraction fixed point theorem combined with resolvent operator to analyze the exact controllability results for fractional neutral integrodifferential systems (FNIDS) with state-dependent delay (SDD) in Banach spaces. An illustration is additionally offered to exhibit the achieved hypotheses.
\end{abstract}

\section{Introduction}

The rise of fractional calculus emerge new inquiries in basic physics, which gives awesome complicated enthusiasm to the mathematicians and physicists in the principle of fractional calculus. The fractional differential equations (FDE) have been viewed as to be the beneficial tool, which can summarize dynamical behavior of real life phenomena more precisely. Case in point, the nonlinear oscillation of earthquake can be effectively displayed with fractional derivatives. We can locate the various uses of FDE in control theory, nonlinear oscillation of earthquake, the fluid-dynamic traffic model, aerodynamics and in nearly every discipline of science and engineering. For

Key Words: Controllability, fractional order differential equations, state-dependent delay, Banach fixed point theorem, resolvent operators, semigroup theory.

2010 Mathematics Subject Classification: Primary 93B05, 34K30, 26A33; Secondary 35R10, 47D06.

Received: 1.07 .2015 .

Revised: 2.08.2015.

Accepted: 3.10 .2015 . 
EXACT CONTROLLABILITY OF FRACTIONAL NEUTRAL INTEGRO-DIFFERENTIAL SYSTEMS WITH STATE-DEPENDENT DELAY IN BANACH SPACES

fundamental certainties about fractional systems, one can make reference to the books $[1,2]$, and the papers $[3,4,5,6,7,8,9]$, and the references cited therein. FDE with delay features happen in several areas such as medical and physical with SDD or non-constant delay. These days, existence and controllability results of mild solutions for such problems became very attractive and several researchers working on it. As of late, few number of papers have been released on the fractional order problems with SDD, see for instance $[10,11,12,13,14,15,16,17,18,19]$.

On the flip side, the idea of controllability has assumed a focal part all through the historical backdrop of cutting edge control theory. This is the qualitative property of control frameworks and is of specific significance in control theory. Numerous dynamical frameworks are such that the control does not influence the complete state of the dynamical framework yet just a piece of it. Then again, frequently in real industrial procedures it is conceivable to notice just a specific piece of the complete state of the dynamical framework. In this way, it is essential to figure out if or not control of the complete state of the dynamical framework is conceivable. In this way, here the idea of complete controllability and approximate controllability emerges. Generally discussing, controllability usually indicates that it is conceivable to steer dynamical framework from a arbitrary beginning state to the coveted last state utilizing the set of acceptable controls.

These days, the controllability for different sorts of fractional differential and integro-differential frameworks in theoretical spaces have created substantial passion among scientists, for instance, see $[20,22,23,24,25,26$, 27, 28, 21]. As of late, Santos et al. [16, 22, 23] researched the existence of solutions for FNIDE with unbounded or SDD delay in Banach spaces. Shu et al. [24] looked into the existence results for FDE with nonlocal conditions of order $\alpha \in(1,2)$. In $[25,26]$, the writers present sufficient conditions for the existence and approximate controllability of fractional order neutral differential and stochastic differential system with infinite delay. Kexue et al. [27] assessed the controllability of nonlocal FDE of order $\alpha \in(1,2]$. Sakthivel et al. [28] identified the approximate controllability of fractional dynamical system by selecting appropriate fixed point theorem whereas Rajivgandhi et al. [29] established the approximate controllability of fractional stochastic integrodifferential equations with infinite delay of order $1<\alpha<2$ by utilizing the Banach contraction principle. Lately, in $[17,18,19]$, the authors discussed the approximate controllability results for FNIDS with SDD by utilizing the suitable fixed point theorem. Having said that, exact controllability results for FNIDS with SDD in $\mathscr{B}_{h}$ phase space adages have not yet been entirely inspected.

Roused by the exertion of the previously stated papers $[14,17,19]$, the 
EXACT CONTROLLABILITY OF FRACTIONAL NEUTRAL INTEGRO-DIFFERENTIAL SYSTEMS WITH STATE-DEPENDENT DELAY IN BANACH SPACES

essential motivation driving this manuscript is to research the controllability of mild solutions for an FNIDS with SDD of the model

$$
\begin{aligned}
D_{t}^{\alpha}[x(t)+ & \left.\mathscr{G}\left(t, x_{\varrho\left(t, x_{t}\right)}, \int_{0}^{t} e_{1}\left(t, s, x_{\varrho\left(s, x_{s}\right)}\right) d s\right)\right]=\mathscr{A} x(t) \\
& +\int_{0}^{t} \mathscr{B}(t-s) x(s) d s+\mathscr{F}\left(t, x_{\varrho\left(t, x_{t}\right)}, \int_{0}^{t} e_{2}\left(t, s, x_{\varrho\left(s, x_{s}\right)}\right) d s\right) \\
& +\mathcal{C} u(t), \quad t \in \mathscr{I}=[0, T], \\
x_{0}= & \varsigma(t) \in \mathscr{B}_{h}, \quad x^{\prime}(0)=0, \quad t \in(-\infty, 0],
\end{aligned}
$$

where the unknown $x(\cdot)$ needs values in the Banach space $\mathbb{X}$ having norm $\|\cdot\|, D_{t}^{\alpha}$ is the Caputo fractional derivative of order $\alpha \in(1,2), \mathscr{A},(\mathscr{B}(t))_{t \geq 0}$ are closed linear operators described on a regular domain which is dense in $(\mathbb{X},\|\cdot\|)$, the control function $u(\cdot) \in L^{2}(\mathscr{I}, U)$, a Banach space of admissible control functions. Moreover, $\mathcal{C}$ is a bounded linear operator from $U$ to $\mathbb{X}$; and $D_{t}^{\alpha} \sigma(t)$ symbolize the Caputo derivative of $\alpha>0$ characterized by

$$
D_{t}^{\alpha} \sigma(t):=\int_{0}^{t} \widetilde{\mu}_{n-\alpha}(t-s) \frac{d^{n}}{d s^{n}} \sigma(s) d s
$$

where $n \geq \alpha$ and $\widetilde{\mu}_{\beta}(t):=\frac{t^{\beta-1}}{\Gamma(\beta)}, t>0, \beta \geq 0$. Further, $\mathscr{G}, \mathscr{F}: \mathscr{I} \times \mathscr{B}_{h} \times \mathbb{X} \rightarrow$ $\mathbb{X}, e_{i}: \mathscr{D} \times \mathscr{B}_{h} \rightarrow \mathbb{X}, i=1,2 ; \mathscr{D}=\{(t, s) \in \mathscr{I} \times \mathscr{I}: 0 \leq s \leq t \leq T\}, \varrho:$ $\mathscr{I} \times \mathscr{B}_{h} \rightarrow(-\infty, T]$ are apposite functions, and $\mathscr{B}_{h}$ is a phase space recognized in Preliminaries.

For almost any continuous function $x$ characterized on $(-\infty, T]$ and any $t \geq 0$, we designate by $x_{t}$ the part of $\mathscr{B}_{h}$ characterized by $x_{t}(\theta)=x(t+\theta)$ for $\theta \leq 0$. Now $x_{t}(\cdot)$ speaks to the historical backdrop of the state from every $\theta \in(-\infty, 0]$ likely the current time $t$.

We proceed ahead as comes after. Section 2 is fully committed to analysis of some vital aspects that will be employed in this work to attain our principal outcomes. In Section 3, we express and demonstrate the controllability outcomes by implies of Banach fixed point theorem. In Section 4, as a final point, a proper case is equipped to reveal the effectiveness of the abstract techniques.

To the best of our understanding, there is no work gave a record of the controllability results for FNIDS with SDD, which is conveyed in the structure (1.1)-(1.2). To pack this crevice, in this manuscript, we mull over this entrancing model.

\section{Preliminaries}

In this part, we present some primary components which are required to 
EXACT CONTROLLABILITY OF FRACTIONAL NEUTRAL INTEGRO-DIFFERENTIAL SYSTEMS WITH STATE-DEPENDENT DELAY IN BANACH SPACES

confirm the principal outcomes.

Let $\mathscr{L}(\mathbb{X})$ symbolizes the Banach space of all bounded linear operators from $\mathbb{X}$ into $\mathbb{X}$ endowed with the uniform operator topology, having its norm recognized as $\|\cdot\|_{\mathscr{L}(\mathbb{X})}$.

Let $C(\mathscr{I}, \mathbb{X})$ symbolize the space of all continuous functions from $\mathscr{I}$ into $\mathbb{X}$, having its norm recognized as $\|\cdot\|_{C(\mathscr{I}, \mathbb{X})}$. Moreover, $B_{r}(x, \mathbb{X})$ symbolizes the closed ball in $\mathbb{X}$ with the middle at $x$ and the distance $r$.

It needs to be outlined that, once the delay is infinite, then we should talk about the theoretical phase space $\mathscr{B}_{h}$ in a beneficial way. In this manuscript, we deliberate phase spaces $\mathscr{B}_{h}$ which are same as described in [30]. So, we bypass the details.

We expect that the phase space $\left(\mathscr{B}_{h},\|\cdot\|_{\mathscr{B}_{h}}\right)$ is a semi-normed linear space of functions mapping $(-\infty, 0]$ into $\mathbb{X}$, and fulfilling the subsequent elementary adages as a result of Hale and Kato ( see case in point in $[31,32]$ ).

If $x:(-\infty, T] \rightarrow \mathbb{X}, T>0$, is continuous on $\mathscr{I}$ and $x_{0} \in \mathscr{B}_{h}$, then for every $t \in \mathscr{I}$ the accompanying conditions hold:

$\left(P_{1}\right) x_{t}$ is in $\mathscr{B}_{h}$;

$\left(P_{2}\right)\|x(t)\|_{\mathbb{X}} \leq H\left\|x_{t}\right\|_{\mathscr{B}_{h}} ;$

$\left(P_{3}\right)\left\|x_{t}\right\|_{\mathscr{B}_{h}} \leq \mathscr{D}_{1}(t) \sup \left\{\|x(s)\|_{\mathbb{X}}: 0 \leq s \leq t\right\}+\mathscr{D}_{2}(t)\left\|x_{0}\right\|_{\mathscr{B}_{h}}$, where $H>0$ is a constant and $\mathscr{D}_{1}(\cdot):[0,+\infty) \rightarrow[0,+\infty)$ is continuous, $\mathscr{D}_{2}(\cdot)$ : $[0,+\infty) \rightarrow[0,+\infty)$ is locally bounded, and $\mathscr{D}_{1}, \mathscr{D}_{2}$ are independent of $x(\cdot)$.

$\left(P_{4}\right)$ The function $t \rightarrow \varsigma_{t}$ is well described and continuous from the set

$$
\mathcal{R}\left(\varrho^{-}\right)=\left\{\varrho(s, \varsigma):(s, \varsigma) \in[0, T] \times \mathscr{B}_{h}\right\},
$$

into $\mathscr{B}_{h}$ and there is a continuous and bounded function $J^{\varsigma}: \mathcal{R}\left(\varrho^{-}\right) \rightarrow$ $(0, \infty)$ to ensure that $\left\|\varsigma_{t}\right\|_{\mathscr{B}_{h}} \leq J^{\varsigma}(t)\|\varsigma\|_{\mathscr{B}_{h}}$ for every $t \in \mathcal{R}\left(\varrho^{-}\right)$.

Recognize the space

$$
\mathscr{B}_{T}=\left\{x:(-\infty, T] \rightarrow \mathbb{X}:\left.x\right|_{\mathscr{I}} \text { is continuous and } x_{0} \in \mathscr{B}_{h}\right\},
$$

where $\left.x\right|_{\mathscr{I}}$ is the constraint of $x$ to the real compact interval on $\mathscr{I}$. The function $\|\cdot\|_{\mathscr{B}_{T}}$ to be a seminorm in $\mathscr{B}_{T}$, it is described by

$$
\|x\|_{\mathscr{B}_{T}}=\|\varsigma\|_{\mathscr{B}_{h}}+\sup \left\{\|x(s)\|_{\mathbb{X}}: s \in[0, T]\right\}, x \in \mathscr{B}_{T} .
$$

Lemma 1. [33, Lemma 2.1] Let $x:(-\infty, T] \rightarrow \mathbb{X}$ be a function in a way that $x_{0}=\varsigma$, and if $\left(P_{4}\right)$ hold, then

$$
\begin{gathered}
\left\|x_{s}\right\|_{\mathscr{B}_{h}} \leq\left(\mathscr{D}_{2}^{*}+J^{\varsigma}\right)\|\varsigma\|_{\mathscr{B}_{h}}+\mathscr{D}_{1}^{*} \sup \left\{\|x(\theta)\|_{\mathbb{X}}: \theta \in[0, \max \{0, s\}]\right\}, \\
s \in \mathcal{R}\left(\varrho^{-}\right) \cup \mathscr{I}
\end{gathered}
$$


EXACT CONTROLLABILITY OF FRACTIONAL NEUTRAL INTEGRO-DIFFERENTIAL SYSTEMS WITH STATE-DEPENDENT DELAY IN BANACH SPACES

where $J^{\varsigma}=\sup _{t \in \mathcal{R}\left(\varrho^{-}\right)} J^{\varsigma}(t), \quad \mathscr{D}_{1}^{*}=\sup _{s \in[0, T]} \mathscr{D}_{1}(s), \quad \mathscr{D}_{2}^{*}=\sup _{s \in[0, T]} \mathscr{D}_{2}(s)$.

To be able to acquire our outcomes, we believe that the subsequent FIDS

$$
\begin{gathered}
D_{t}^{\alpha} x(t)=\mathscr{A} x(t)+\int_{0}^{t} \mathscr{B}(t-s) x(s) d s, \\
x(0)=\varsigma \in \mathbb{X}, \quad x^{\prime}(0)=0,
\end{gathered}
$$

has an associated $\alpha$-resolvent operator of bounded linear operators $\left(\mathcal{R}_{\alpha}(t)\right)_{t \geq 0}$ on $\mathbb{X}$.

Definition 2.1. [22, Definition 2.1] A one parameter family of bounded linear operators $\left(\mathcal{R}_{\alpha}(t)\right)_{t \geq 0}$ on $\mathbb{X}$ is called a $\alpha$-resolvent operator of (2.1)-(2.2) if the subsequent conditions are fulfilled.

(a) The function $\mathcal{R}_{\alpha}(\cdot):[0, \infty) \rightarrow \mathscr{L}(\mathbb{X})$ is strongly continuous and $\mathcal{R}_{\alpha}(0) x=x$ for all $x \in \mathbb{X}$ and $\alpha \in(1,2)$.

(b) For $x \in D(\mathscr{A}), \mathcal{R}_{\alpha}(\cdot) x \in C([0, \infty),[D(\mathscr{A})]) \bigcap C^{1}((0, \infty), \mathbb{X})$, and for every $t \geq 0$, we receive

$$
\begin{aligned}
& D_{t}^{\alpha} \mathcal{R}_{\alpha}(t) x=\mathscr{A} \mathcal{R}_{\alpha}(t) x+\int_{0}^{t} \mathscr{B}(t-s) \mathcal{R}_{\alpha}(s) x d s, \\
& D_{t}^{\alpha} \mathcal{R}_{\alpha}(t) x=\mathcal{R}_{\alpha}(t) \mathscr{A} x+\int_{0}^{t} \mathcal{R}_{\alpha}(t-s) \mathscr{B}(s) x d s .
\end{aligned}
$$

The existence of a $\alpha$-resolvent operator for the model (2.1)-(2.2) was analyzed in [20]. To be able to research our model, we need to consider the conditions $(P 1)-(P 3)$ which are same as stated in [22], consequently we preclude it.

In view of the conditions $(P 1)-(P 3)$, we determine the operator family $\left(\mathcal{R}_{\alpha}(t)\right)_{t \geq 0}$ by

$$
\mathcal{R}_{\alpha}(t)= \begin{cases}\frac{1}{2 \pi i} \int_{\Gamma_{r, \theta}} e^{\lambda t} G_{\alpha}(\lambda) d \lambda, & t>0, \\ I, & t=0,\end{cases}
$$

where

$$
\rho_{\alpha}\left(G_{\alpha}\right)=\left\{\lambda \in \mathbb{C}: G_{\alpha}(\lambda):=\lambda^{\alpha-1}\left(\lambda^{\alpha} I-\mathscr{A}-\widehat{\mathscr{B}}(\lambda)\right)^{-1} \in \mathscr{L}(\mathbb{X})\right\} .
$$

Hereafter, we expect that the conditions $(P 1)-(P 3)$ are fulfilled. Further, we need to talk about the mild solution for the model (1.1)-(1.2). For this 
EXACT CONTROLLABILITY OF FRACTIONAL NEUTRAL INTEGRO-DIFFERENTIAL SYSTEMS WITH STATE-DEPENDENT DELAY IN BANACH SPACES

intent, it is necessary to discuss the subsequent non-homogeneous model

$$
\begin{gathered}
D_{t}^{\alpha} x(t)=\mathscr{A} x(t)+\int_{0}^{t} \mathscr{B}(t-s) x(s) d s+\mathscr{F}(t), \quad t \in \mathscr{I}, \\
x(0)=x_{0}, \quad x^{\prime}(0)=0,
\end{gathered}
$$

where $\alpha \in(1,2)$ and $\mathscr{F} \in L^{1}(\mathscr{I}, \mathbb{X})$.

Definition 2.2 ([22, Definition 2.6]). Let $\alpha \in(1,2)$, we describe the family $\left(\mathcal{S}_{\alpha}(t)\right)_{t \geq 0}$ by

$$
\mathcal{S}_{\alpha}(t) x:=\int_{0}^{t} \widetilde{\mu}_{\alpha-1}(t-s) \mathcal{R}_{\alpha}(s) x d s
$$

for each $t \geq 0$.

The properties and auxiliary results of (2.1)-(2.2) and (2.6)-(2.7) are broadly examined in $[20,22]$. To maintain a strategic distance from the redundancy, here we discard it.

In the subsequent result, we signify by $(-\mathscr{A})^{\vartheta}$ the fractional power of the operator $-\mathscr{A}$, (see [34] for details).

Lemma 2 ([22, Lemma 3.1]). Suppose that the conditions (P1)-(P3) are satisfied. Let $\alpha \in(1,2)$ and $\vartheta \in(0,1)$ such that $\alpha \vartheta \in(0,1)$, then there exists positive number $C$ such that

$$
\begin{gathered}
\left\|(-\mathscr{A})^{\vartheta} \mathcal{R}_{\alpha}(t)\right\| \leq C e^{r t} t^{-\alpha \vartheta}, \\
\left\|(-\mathscr{A})^{\vartheta} \mathcal{S}_{\alpha}(t)\right\| \leq C e^{r t} t^{\alpha(1-\vartheta)-1},
\end{gathered}
$$

for all $t>0$. If $x \in\left[D\left((-\mathscr{A})^{\vartheta}\right)\right]$, then

$$
(-\mathscr{A})^{\vartheta} \mathcal{R}_{\alpha}(t) x=\mathcal{R}_{\alpha}(t)(-\mathscr{A})^{\vartheta} x, \quad(-\mathscr{A})^{\vartheta} \mathcal{S}_{\alpha}(t) x=\mathcal{S}_{\alpha}(t)(-\mathscr{A})^{\vartheta} x .
$$

Definition 2.3. Let $x_{T}(\varsigma ; u)$ be the state value of the model (1.1)-(1.2) at terminal time $T$ corresponding to the control $u$ and the initial value $\varsigma \in \mathscr{B}_{h}$. Present the set $\mathscr{R}(T, \varsigma)=\left\{x_{T}(\varsigma ; u)(0): u(\cdot) \in L^{2}(\mathscr{I}, U)\right\}$, which is known as the reachable set of model (1.1)-(1.2) at terminal time $T$.

Definition 2.4. The model (1.1)-(1.2) is said to be exactly controllable on $\mathscr{I}$ if $\mathscr{R}(T, \varsigma)=\mathbb{X}$.

Assume that the fractional differential control model

$$
\begin{aligned}
D^{\alpha} x(t) & =\mathscr{A} x(t)+(\mathcal{C} u)(t), \quad t \in \mathscr{I}, \\
x_{0} & =\varsigma \in \mathscr{B}_{h},
\end{aligned}
$$


EXACT CONTROLLABILITY OF FRACTIONAL NEUTRAL INTEGRO-DIFFERENTIAL SYSTEMS WITH STATE-DEPENDENT DELAY IN BANACH SPACES

is exactly controllable. It is practical at this position to present the controllability operator linked with (2.11)-(2.12) as

$$
\Gamma_{0}^{T}=\int_{0}^{T} \mathcal{S}_{\alpha}(T-s) \mathcal{C}^{*} \mathcal{S}_{\alpha}^{*}(T-s) d s,
$$

where $\mathcal{C}^{*}$ and $\mathcal{S}_{\alpha}^{*}(t)$ denotes the adjoints of $\mathcal{C}$ and $\mathcal{S}_{\alpha}(t)$, accordingly. It is simple that the operator $\Gamma_{0}^{T}$ is a linear bounded operator [35, Theorem 3.2].

Lemma 3. If the linear fractional model (2.11)-(2.12) is exactly controllable if and only then for some $\gamma>0$ such that $\left\langle\Gamma_{0}^{T} x, x\right\rangle \geq \gamma\|x\|^{2}$, for all $x \in \mathbb{X}$ and as a result $\left\|\left(\Gamma_{0}^{T}\right)^{-1}\right\| \leq \frac{1}{\gamma}$.

Remark 2.1. Further, we assume that the linear fractional control system (2.11)-(2.12) is exactly controllable.

\section{Controllability Results}

In this segment, we present and demonstrate the exact controllability results for the structure (1.1)-(1.2) under Banach fixed point theorem. Initially, we present the mild solution for the model (1.1)-(1.2).

Definition 3.1. [22, Definition 3.4] A function $x:(-\infty, T] \rightarrow \mathbb{X}$, is called a mild solution of (1.1)-(1.2) on $[0, T]$, if $x_{0}=\varsigma ;\left.x\right|_{[0, T]} \in C([0, T]: \mathbb{X})$; the function $s \rightarrow \mathscr{A} \mathcal{S}_{\alpha}(t-s) \mathscr{G}\left(s, x_{\varrho\left(s, x_{s}\right)}, \int_{0}^{s} e_{1}\left(s, \tau, x_{\varrho\left(\tau, x_{\tau}\right)}\right) d \tau\right)$ and $s \rightarrow$ $\int_{0}^{s} \mathscr{B}(s-\tau) \mathcal{S}_{\alpha}(t-s) \mathscr{G}\left(\tau, x_{\varrho\left(\tau, x_{\tau}\right)}, \int_{0}^{\tau} e_{1}\left(\tau, \xi, x_{\varrho\left(\xi, x_{\xi}\right)}\right) d \xi\right) d \tau$ is integrable on $[0, t)$ for all $t \in(0, T]$ and for $t \in[0, T]$,

$$
\begin{aligned}
x(t)= & \mathcal{R}_{\alpha}(t)[\varsigma(0)+\mathscr{G}(0, \varsigma(0), 0)]-\mathscr{G}\left(t, x_{\varrho\left(t, x_{t}\right)}, \int_{0}^{t} e_{1}\left(t, s, x_{\varrho\left(s, x_{s}\right)}\right) d s\right) \\
& -\int_{0}^{t} \mathscr{A} \mathcal{S}_{\alpha}(t-s) \mathscr{G}\left(s, x_{\varrho\left(s, x_{s}\right)}, \int_{0}^{s} e_{1}\left(s, \tau, x_{\varrho\left(\tau, x_{\tau}\right)}\right) d \tau\right) d s \\
& -\int_{0}^{t} \int_{0}^{s} \mathscr{B}(s-\tau) \mathcal{S}_{\alpha}(t-s) \mathscr{G}\left(\tau, x_{\varrho\left(\tau, x_{\tau}\right)}, \int_{0}^{\tau} e_{1}\left(\tau, \xi, x_{\varrho\left(\xi, x_{\xi}\right)}\right) d \xi\right) d \tau d s \\
& +\int_{0}^{t} \mathcal{S}_{\alpha}(t-s) \mathscr{F}\left(s, x_{\varrho\left(s, x_{s}\right)}, \int_{0}^{s} e_{2}\left(s, \tau, x_{\varrho\left(\tau, x_{\tau}\right)}\right) d \tau\right) d s \\
& +\int_{0}^{t} \mathcal{S}_{\alpha}(t-s) \mathcal{C} u(s) d s .
\end{aligned}
$$

Presently, we itemizing the subsequent suppositions: 
(H1) The operator families $\mathcal{R}_{\alpha}(t)$ and $\mathcal{S}_{\alpha}(t)$ are compact for all $t>0$, and there exists a constant $\mathcal{M}$ in a way that $\left\|\mathcal{R}_{\alpha}(t)\right\|_{\mathscr{L}(\mathbb{X})} \leq \mathcal{M}$ and $\left\|\mathcal{S}_{\alpha}(t)\right\|_{\mathscr{L}(\mathbb{X})} \leq \mathcal{M}$ for every $t \in \mathscr{I}$ and

$$
\left\|(-\mathscr{A})^{\vartheta} \mathcal{S}_{\alpha}(t)\right\|_{\mathbb{X}} \leq \mathcal{M} t^{\alpha(1-\vartheta)-1}, \quad 0<t \leq T .
$$

(H2) The subsequent conditions are fulfilled.

(a) $\mathscr{B}(\cdot) x \in C(\mathscr{I}, \mathbb{X})$ for every $x \in\left[D\left((-\mathscr{A})^{1-\vartheta}\right)\right]$.

(b) There is a function $\mu(\cdot) \in L^{1}\left(\mathscr{I} ; \mathbb{R}^{+}\right)$, to ensure that

$$
\left\|\mathscr{B}(s) \mathcal{S}_{\alpha}(t)\right\|_{\mathscr{L}\left(\left[D\left((-\mathscr{A})^{\vartheta}\right)\right], \mathbb{X}\right)} \leq \mathcal{M} \mu(s) t^{\alpha \vartheta-1}, \quad 0 \leq s<t \leq T .
$$

(H3) The function $\mathscr{F}: \mathscr{I} \times \mathscr{B}_{h} \times \mathbb{X} \rightarrow \mathbb{X}$ is continuous and we can find positive constants $L_{\mathscr{F}}, \widetilde{L}_{\mathscr{F}}>0$ and $L_{\mathscr{F}}^{*}>0$ in ways that for all $t \in \mathscr{I}, x, y \in \mathbb{X}$,

$$
\left\|\mathscr{F}\left(t, \psi_{1}, x\right)-\mathscr{F}\left(t, \psi_{2}, y\right)\right\|_{\mathbb{X}} \leq L_{\mathscr{F}}\left\|\psi_{1}-\psi_{2}\right\|_{\mathscr{B}_{h}}+\widetilde{L}_{\mathscr{F}}\|x-y\|_{\mathbb{X}},
$$

and

$$
L_{\mathscr{F}}^{*}=\max _{t \in \mathscr{I}}\|\mathscr{F}(t, 0,0)\|_{\mathbb{X}}
$$

(H4) $e_{i}: \mathscr{D} \times \mathscr{B}_{h} \rightarrow \mathbb{X}$ is continuous and we can find constants $L_{e_{i}}>0, L_{e_{i}}^{*}>0$ to ensure that for all $(t, s) \in \mathscr{D},(\varsigma, \psi) \in \mathscr{B}_{h}^{2}, i=1,2$;

$$
\left\|e_{i}(t, s, \varsigma)-e_{i}(t, s, \psi)\right\|_{\mathbb{X}} \leq L_{e_{i}}\|\varsigma-\psi\|_{\mathscr{B}_{h}}
$$

and

$$
L_{e_{i}}^{*}=\max _{t \in \mathscr{I}}\left\|e_{i}(t, s, 0)\right\|_{\mathbb{X}}, \quad i=1,2
$$

(H5) The function $\mathscr{G}(\cdot)$ is $(-\mathscr{A})^{\vartheta}$-valued, $\mathscr{G}: \mathscr{I} \times \mathscr{B}_{h} \times \mathbb{X} \rightarrow\left[D\left((-\mathscr{A})^{-\vartheta}\right)\right]$ is continuous and there exist positive constants $L_{\mathscr{G}}, \widetilde{L}_{\mathscr{G}}>0$ and $L_{\mathscr{G}}^{*}>0$ such that for all $\left(t, \varsigma_{j}\right) \in \mathscr{I} \times \mathscr{B}_{h}, j=1,2 ; x, y \in \mathbb{X}$,

$$
\begin{gathered}
\left\|(-\mathscr{A})^{\vartheta} \mathscr{G}\left(t, \varsigma_{1}, x\right)-(-\mathscr{A})^{\vartheta} \mathscr{G}\left(t, \varsigma_{2}, y\right)\right\|_{\mathbb{X}} \leq L_{\mathscr{G}}\left\|\varsigma_{1}-\varsigma_{2}\right\|_{\mathscr{B}_{h}}+\widetilde{L}_{\mathscr{G}}\|x-y\|_{\mathbb{X}}, \\
\left\|(-\mathscr{A})^{\vartheta} \mathscr{G}(t, \varsigma, 0)\right\|_{\mathbb{X}} \leq L \mathscr{G}\|\varsigma\|_{\mathscr{B}_{h}}+L_{\mathscr{G}}^{*},
\end{gathered}
$$

where

$$
L_{\mathscr{G}}^{*}=\max _{t \in \mathscr{I}}\left\|(-\mathscr{A})^{\vartheta} \mathscr{G}(t, 0,0)\right\|_{\mathbb{X}}
$$

(H6) The following inequalities holds: 
(i) Let

$$
\begin{aligned}
& \left(\frac{1}{\gamma} \mathcal{M}^{2} \mathcal{M}_{\mathcal{C}}^{2} T\right)\left\|x_{T}\right\|+\left(1+\frac{1}{\gamma} \mathcal{N}^{2} \mathcal{M}_{\mathfrak{C}}^{2} T\right)\left[\mathcal{N M}_{0} L_{\mathscr{G}}\left\|_{\varsigma}\right\|_{\mathscr{B}_{h}}+\mathcal{M N}_{0} L_{\mathscr{G}}^{*}\right. \\
& +\left(L_{\mathscr{G}}^{*}+\widetilde{L}_{\mathscr{G}} T L_{e_{1}}^{*}\right)\left\{\mathcal{M}_{0}+\frac{\mathcal{N} T^{\alpha \vartheta}}{\alpha \vartheta}\left(1+\int_{0}^{T} \mu(\tau) d \tau\right)\right\} \\
& +\mathcal{N} T\left(L_{\mathscr{F}}^{*}+\widetilde{L}_{\mathscr{F}} T L_{e_{2}}^{*}\right)+\left(\mathscr{D}_{1}^{*} r+c_{n}\right)\left[\mathcal{M} T\left(L_{\mathscr{F}}+\widetilde{L}_{\mathscr{F}} T L_{e_{2}}\right)\right. \\
& \left.\left.\quad+\left\{\mathcal{M}_{0}+\frac{\mathcal{N} T^{\alpha \vartheta}}{\alpha \vartheta}\left(1+\int_{0}^{T} \mu(\tau) d \tau\right)\right\}\left(L \mathscr{G}+\widetilde{L}_{\mathscr{G}} T L_{e_{1}}\right)\right]\right] \leq r,
\end{aligned}
$$

for some $r>0$.

(ii) Let

$$
\begin{aligned}
\Lambda= & \left(1+\frac{1}{\gamma} \mathcal{M}^{2} \mathcal{M}_{\mathcal{C}}^{2} T\right) \mathscr{D}_{1}^{*}\left[\mathcal{N} T\left(L_{\mathscr{F}}+\widetilde{L}_{\mathscr{F}} T L_{e_{2}}\right)\right. \\
& \left.+\left\{\mathcal{M}_{0}+\frac{\mathcal{M} T^{\alpha \vartheta}}{\alpha \vartheta}\left(1+\int_{0}^{T} \mu(\tau) d \tau\right)\right\}\left(L_{\mathscr{G}}+\widetilde{L}_{\mathscr{G}} T L_{e_{1}}\right)\right]<1
\end{aligned}
$$

be such that $0 \leq \Lambda<1$.

Theorem 3.1. Assume that the conditions (H1)-(H6) hold. Then the control system (1.1)-(1.2) is exactly controllable on $\mathscr{I}$.

Proof. Utilizing the hypothesis, for an arbitrary function $x(\cdot)$, choose the feedback control function as follows:

$$
\begin{aligned}
u_{x}(t)= & \mathcal{C}^{*} \mathcal{S}_{\alpha}^{*}(T-t)\left(\Gamma_{0}^{T}\right)^{-1}\left[x_{T}-\mathcal{R}_{\alpha}(T)[\varsigma(0)+\mathscr{G}(0, \varsigma(0), 0)]\right. \\
& +\mathscr{G}\left(T, x_{\varrho\left(T, x_{T}\right)}, \int_{0}^{T} e_{1}\left(T, s, x_{\varrho\left(s, x_{s}\right)}\right) d s\right) \\
& +\int_{0}^{T} \mathscr{A} \mathcal{S}_{\alpha}(T-s) \mathscr{G}\left(s, x_{\varrho\left(s, x_{s}\right)}, \int_{0}^{s} e_{1}\left(s, \tau, x_{\varrho\left(\tau, x_{\tau}\right)}\right) d \tau\right) d s \\
& +\int_{0}^{T} \int_{0}^{s} \mathscr{B}(s-\tau) \mathcal{S}_{\alpha}(T-s) \\
& (\times) \mathscr{G}\left(\tau, x_{\varrho\left(\tau, x_{\tau}\right)}, \int_{0}^{\tau} e_{1}\left(\tau, \xi, x_{\varrho\left(\xi, x_{\xi}\right)}\right) d \xi\right) d \tau d s
\end{aligned}
$$




$$
\left.-\int_{0}^{T} \mathcal{S}_{\alpha}(T-s) \mathscr{F}\left(s, x_{\varrho\left(s, x_{s}\right)}, \int_{0}^{s} e_{2}\left(s, \tau, x_{\varrho\left(\tau, x_{\tau}\right)}\right) d \tau\right) d s\right]
$$

Presently, we determine the operator $\Upsilon: \mathscr{B}_{T} \rightarrow \mathscr{B}_{T}$ by

$$
(\Upsilon x)(t)=\left\{\begin{aligned}
& \mathcal{R}_{\alpha}(t)[\varsigma(0)+\mathscr{G}(0, \varsigma(0), 0)] \\
&- \mathscr{G}\left(t, x_{\varrho\left(t, x_{t}\right)}, \int_{0}^{t} e_{1}\left(t, s, x_{\varrho\left(s, x_{s}\right)}\right) d s\right) \\
&-\int_{0}^{t} \mathscr{A} \mathcal{S}_{\alpha}(t-s) \mathscr{G}\left(s, x_{\varrho\left(s, x_{s}\right)}, \int_{0}^{s} e_{1}\left(s, \tau, x_{\varrho\left(\tau, x_{\tau}\right)}\right) d \tau\right) d s \\
&-\int_{0}^{t} \int_{0}^{s} \mathscr{B}(s-\tau) \mathcal{S}_{\alpha}(t-s) \\
&(\times) \mathscr{G}\left(\tau, x_{\varrho\left(\tau, x_{\tau}\right)}, \int_{0}^{\tau} e_{1}\left(\tau, \xi, x_{\varrho\left(\xi, x_{\xi}\right)}\right) d \xi\right) d \tau d s \\
&+\int_{0}^{t} \mathcal{S}_{\alpha}(t-s) \mathscr{F}\left(s, x_{\varrho\left(s, x_{s}\right)}, \int_{0}^{s} e_{2}\left(s, \tau, x_{\varrho\left(\tau, x_{\tau}\right)}\right) d \tau\right) d s \\
&+\int_{0}^{t} \mathcal{S}_{\alpha}(t-s) \mathcal{C} u_{x}(s) d s, \quad t \in \mathscr{I} .
\end{aligned}\right.
$$

Observe that the control (3.2) transfers the system (1.1)-(1.2) from the initial state $\varsigma$ to the final state $x_{T}$ provided that the operator $\Upsilon$ has a fixed point. To confirm the exact controllability outcome, it is adequate to demonstrate that the operator $\Upsilon$ has a fixed point in $\mathscr{B}_{T}$.

We express the function $y(\cdot):(-\infty, T] \rightarrow \mathbb{X}$ by

$$
y(t)=\left\{\begin{array}{l}
\varsigma(t), \quad t \leq 0 \\
\mathcal{R}_{\alpha}(t) \varsigma(0), \quad t \in \mathscr{I}
\end{array}\right.
$$

then $y_{0}=\varsigma$. For every function $z \in C(\mathscr{I}, \mathbb{R})$ with $z(0)=0$, we allocate as $\bar{z}$ is characterized by

$$
\bar{z}(t)= \begin{cases}0, & t \leq 0 \\ z(t), & t \in \mathscr{I}\end{cases}
$$

If $x(\cdot)$ fulfills $(3.1)$, we are able to split it as $x(t)=y(t)+z(t), t \in \mathscr{I}$, which suggests $x_{t}=y_{t}+z_{t}$, for each $t \in \mathscr{I}$ and also the function $z(\cdot)$ fulfills

$$
\begin{aligned}
z(t)= & \mathcal{R}_{\alpha}(t) \mathscr{G}(0, \varsigma, 0)-\mathscr{G}\left(t, z_{\varrho\left(t, z_{t}+y_{t}\right)}+y_{\varrho\left(t, z_{t}+y_{t}\right)},\right. \\
& \left.\int_{0}^{t} e_{1}\left(t, s, z_{\varrho\left(s, z_{s}+y_{s}\right)}+y_{\varrho\left(s, z_{s}+y_{s}\right)}\right) d s\right)
\end{aligned}
$$


EXACT CONTROLLABILITY OF FRACTIONAL NEUTRAL INTEGRO-DIFFERENTIAL SYSTEMS WITH STATE-DEPENDENT DELAY IN BANACH SPACES

$$
\begin{aligned}
- & \int_{0}^{t} \mathscr{A} \mathcal{S}_{\alpha}(t-s) \mathscr{G}\left(s, z_{\varrho\left(s, z_{s}+y_{s}\right)}+y_{\varrho\left(s, z_{s}+y_{s}\right)},\right. \\
& \left.\int_{0}^{s} e_{1}\left(s, \tau, z_{\varrho\left(\tau, z_{\tau}+y_{\tau}\right)}+y_{\varrho\left(\tau, z_{\tau}+y_{\tau}\right)}\right) d \tau\right) d s \\
- & \int_{0}^{t} \int_{0}^{s} \mathscr{B}(s-\tau) \mathcal{S}_{\alpha}(t-s) \mathscr{G}\left(\tau, z_{\varrho\left(\tau, z_{\tau}+y_{\tau}\right)}+y_{\varrho\left(\tau, z_{\tau}+y_{\tau}\right)},\right. \\
& \left.\int_{0}^{\tau} e_{1}\left(\tau, \xi, z_{\varrho\left(\xi, z_{\xi}+y_{\xi}\right)}+y_{\varrho\left(\xi, z_{\xi}+y_{\xi}\right)}\right) d \xi\right) d \tau d s \\
+ & \int_{0}^{t} \mathcal{S}_{\alpha}(t-s) \mathscr{F}\left(s, z_{\varrho\left(s, z_{s}+y_{s}\right)}+y_{\varrho\left(s, z_{s}+y_{s}\right)},\right. \\
& \left.\int_{0}^{s} e_{2}\left(s, \tau, z_{\varrho\left(\tau, z_{\tau}+y_{\tau}\right)}+y_{\varrho\left(\tau, z_{\tau}+y_{\tau}\right)}\right) d \tau\right) d s \\
+ & \int_{0}^{t} \mathcal{S}_{\alpha}(t-s) \mathcal{C} u_{z+y}(s) d s,
\end{aligned}
$$

where

$$
u_{z+y}(t)=\left\{\begin{array}{l}
\mathcal{Q}^{*} \mathcal{S}_{\alpha}^{*}(T-t)\left(\Gamma_{0}^{T}\right)^{-1}\left[x_{T}-\mathcal{R}_{\alpha}(T) \mathscr{G}(0, \varsigma, 0)\right. \\
+\mathscr{G}\left(T, z_{\varrho\left(T, z_{T}+y_{T}\right)}+y_{\varrho\left(T, z_{T}+y_{T}\right)}\right. \\
\left.\int_{0}^{T} e_{1}\left(T, s, z_{\varrho\left(s, z_{s}+y_{s}\right)}+y_{\varrho\left(s, z_{s}+y_{s}\right)}\right) d s\right) \\
+\int_{0}^{s} \mathscr{A}_{\alpha}(T-s) \mathscr{G}\left(s, z_{\varrho\left(s, z_{s}+y_{s}\right)}+y_{\varrho}\left(s, z_{s}+y_{s}\right)\right. \\
\left.\quad e_{0}\left(s, \tau, z_{\varrho\left(\tau, z_{\tau}+y_{\tau}\right)}+y_{\varrho\left(\tau, z_{\tau}+y_{\tau}\right)}\right) d \tau\right) d s \\
+\int_{0}^{T} \int_{0}^{s} \mathscr{B}(s-\tau) \mathcal{S}_{\alpha}(T-s) \mathscr{G}_{(\tau,}\left(\tau, z_{\varrho\left(\tau, z_{\tau}+y_{\tau}\right)}+y_{\varrho\left(\tau, z_{\tau}+y_{\tau}\right)},\right. \\
\left.\int_{0}^{\tau} e_{1}\left(\tau, \xi, z_{\varrho\left(\xi, z_{\xi}+y_{\xi}\right)}+y_{\varrho\left(\xi, z_{\xi}+y_{\xi}\right)}\right) d \xi\right) d \tau d s \\
-\int_{0}^{T} \mathcal{S}_{\alpha}(T-s) \mathscr{F}\left(s, z_{\varrho\left(s, z_{s}+y_{s}\right)}+y_{\varrho\left(s, z_{s}+y_{s}\right)}\right. \\
\left.\left.\int_{0}^{s} e_{2}\left(s, \tau, z_{\varrho\left(\tau, z_{\tau}+y_{\tau}\right)}+y_{\varrho\left(\tau, z_{\tau}+y_{\tau}\right)}\right) d \tau\right) d s\right]
\end{array}\right.
$$

Let $\mathscr{B}_{T}^{0}=\left\{z \in \mathscr{B}_{T}^{0}: z_{0}=0 \in \mathscr{B}_{h}\right\}$. Let $\|\cdot\|_{\mathscr{B}_{T}^{0}}$ be the seminorm in $\mathscr{B}_{T}^{0}$ described by

$$
\|z\|_{\mathscr{B}_{T}^{0}}=\sup _{t \in \mathscr{I}}\|z(t)\|_{\mathbb{X}}+\left\|z_{0}\right\|_{\mathscr{B}_{h}}=\sup _{t \in \mathscr{I}}\|z(t)\|_{\mathbb{X}}, \quad z \in \mathscr{B}_{T}^{0}
$$


EXACT CONTROLLABILITY OF FRACTIONAL NEUTRAL INTEGRO-DIFFERENTIAL SYSTEMS WITH STATE-DEPENDENT DELAY IN BANACH SPACES

as a result $\left(\mathscr{B}_{T}^{0},\|\cdot\|_{\mathscr{B}_{T}^{0}}\right)$ is a Banach space. We delimit the operator $\bar{\Upsilon}: \mathscr{B}_{T}^{0} \rightarrow \mathscr{B}_{T}^{0}$ by

$$
(\bar{\Upsilon} z)(t)=\left\{\begin{array}{l}
\mathcal{R}_{\alpha}(t) \mathscr{G}(0, \varsigma, 0)-\mathscr{G}\left(t, z_{\varrho\left(t, z_{t}+y_{t}\right)}+y_{\varrho\left(t, z_{t}+y_{t}\right)}\right. \\
\left.\int_{0}^{t} e_{1}\left(t, s, z_{\varrho\left(s, z_{s}+y_{s}\right)}+y_{\varrho\left(s, z_{s}+y_{s}\right)}\right) d s\right) \\
-\int_{0}^{t} \mathscr{A}_{\alpha}(t-s) \mathscr{G}\left(s, z_{\varrho\left(s, z_{s}+y_{s}\right)}+y_{\varrho\left(s, z_{s}+y_{s}\right)}\right. \\
\left.\int_{0}^{s} e_{1}\left(s, \tau, z_{\varrho\left(\tau, z_{\tau}+y_{\tau}\right)}+y_{\varrho\left(\tau, z_{\tau}+y_{\tau}\right)}\right) d \tau\right) d s \\
-\int_{0}^{t} \int_{0}^{s} \mathscr{B}(s-\tau) \mathcal{S}_{\alpha}(t-s) \mathscr{G}\left(\tau, z_{\varrho}\left(\tau, z_{\tau}+y_{\tau}\right)+y_{\varrho\left(\tau, z_{\tau}+y_{\tau}\right)}\right. \\
\left.\int_{0}^{\tau} e_{1}\left(\tau, \xi, z_{\varrho\left(\xi, z_{\xi}+y_{\xi}\right)}+y_{\varrho\left(\xi, z_{\xi}+y_{\xi}\right)}\right) d \xi\right) d \tau d s \\
+\int_{0}^{t} \mathcal{S}_{\alpha}(t-s) \mathscr{F}\left(s, z_{\varrho}\left(s, z_{s}+y_{s}\right)+y_{\varrho}\left(s, z_{s}+y_{s}\right)\right. \\
\left.\int_{0}^{s} e_{2}\left(s, \tau, z_{\varrho\left(\tau, z_{\tau}+y_{\tau}\right)}+y_{\varrho\left(\tau, z_{\tau}+y_{\tau}\right)}\right) d \tau\right) d s \\
+\int_{0}^{t} \mathcal{S}_{\alpha}(t-s) \mathcal{C} u_{z+y}(s) d s .
\end{array}\right.
$$

Remark 3.1. Let $B_{r}=\{x \in \mathbb{X}:\|x\| \leq r\}$ for some $r>0$. From the above discussion, we have the subsequent estimates:

(i)

$$
\begin{aligned}
& \left\|z_{\varrho\left(s, z_{s}+y_{s}\right)}+y_{\varrho\left(s, z_{s}+y_{s}\right)}\right\|_{\mathscr{B}_{h}} \\
& \leq\left\|z_{\varrho\left(s, z_{s}+y_{s}\right)}\right\| \mathscr{B}_{h}+\left\|y_{\varrho\left(s, z_{s}+y_{s}\right)}\right\|_{\mathscr{B}_{h}} \\
& \leq \mathscr{D}_{1}^{*} \sup _{0 \leq \tau \leq s}\|z(\tau)\|_{\mathbb{X}}+\left(\mathscr{D}_{2}^{*}+J^{\varsigma}\right)\left\|z_{0}\right\|_{\mathscr{B}_{h}}+\mathscr{D}_{1}^{*}|y(s)|+\left(\mathscr{D}_{2}^{*}+J^{\varsigma}\right)\left\|y_{0}\right\|_{\mathscr{B}_{h}} \\
& \leq \mathscr{D}_{1}^{*} \sup _{0 \leq \tau \leq s}\|z(\tau)\|_{\mathbb{X}}+\mathscr{D}_{1}^{*}\left\|\mathcal{R}_{\alpha}(t)\right\|_{\mathscr{L}(\mathbb{X})}|\varsigma(0)|+\left(\mathscr{D}_{2}^{*}+J^{\varsigma}\right)\|\varsigma\|_{\mathscr{B}_{h}} \\
& \leq \mathscr{D}_{1}^{*} \sup _{0 \leq \tau \leq s}\|z(\tau)\|_{\mathbb{X}}+\mathscr{D}_{1}^{*} \mathcal{M} H\|\varsigma\|_{\mathscr{B}_{h}}+\left(\mathscr{D}_{2}^{*}+J^{\varsigma}\right)\|\varsigma\|_{\mathscr{B}_{h}} \\
& \leq \mathscr{D}_{1}^{*} \sup _{0 \leq \tau \leq s}\|z(\tau)\|_{\mathbb{X}}+\left(\mathscr{D}_{1}^{*} \mathcal{M} H+\mathscr{D}_{2}^{*}+J^{\varsigma}\right)\|\varsigma\|_{\mathscr{B}_{h}} .
\end{aligned}
$$

In the event that $\|z\|_{\mathbb{X}}<r, r>0$, then

$$
\left\|z_{\rho\left(s, z_{s}+y_{s}\right)}+y_{\rho\left(s, z_{s}+y_{s}\right)}\right\|_{\mathscr{B}_{h}} \leq \mathscr{D}_{1}^{*} r+c_{n},
$$

where $c_{n}=\left(\mathscr{D}_{1}^{*} \mathcal{M} H+\mathscr{D}_{2}^{*}+J^{\varsigma}\right)\|\varsigma\|_{\mathscr{B}_{h}}$. 
EXACT CONTROLLABILITY OF FRACTIONAL NEUTRAL INTEGRO-DIFFERENTIAL SYSTEMS WITH STATE-DEPENDENT DELAY IN BANACH SPACES

(ii) From suppositions (H1) and (H5), we sustain

$$
\left\|\mathcal{R}_{\alpha}(t)\right\|_{\mathscr{L}(\mathbb{X})}\|\mathscr{G}(0, \varsigma, 0)\|_{\mathbb{X}} \leq \mathcal{M} \mathcal{M}_{0}\left[L_{\mathscr{G}}\left\|_{\varsigma}\right\|_{\mathscr{B}_{h}}+L_{\mathscr{G}}^{*}\right],
$$

where $\left\|(-\mathscr{A})^{-\vartheta}\right\|=\mathcal{M}_{0}$.

(iii)

$$
\begin{aligned}
& \left\|\mathscr{G}\left(t, z_{\varrho\left(t, z_{t}+y_{t}\right)}+y_{\varrho\left(t, z_{t}+y_{t}\right)}, \int_{0}^{t} e_{1}\left(t, s, z_{\varrho\left(s, z_{s}+y_{s}\right)}+y_{\varrho\left(s, z_{s}+y_{s}\right)}\right) d s\right)\right\|_{\mathbb{X}} \\
& \leq\left\|(-\mathscr{A})^{-\vartheta}\right\|\left[\|(-\mathscr{A})^{\vartheta} \mathscr{G}\left(t, z_{\varrho\left(t, z_{t}+y_{t}\right)}+y_{\varrho\left(t, z_{t}+y_{t}\right)},\right.\right. \\
& \left.\int_{0}^{t} e_{1}\left(t, s, z_{\varrho\left(s, z_{s}+y_{s}\right)}+y_{\varrho\left(s, z_{s}+y_{s}\right)}\right) d s\right)-(-\mathscr{A})^{\vartheta} \mathscr{G}(t, 0,0) \|_{\mathbb{X}} \\
& \left.+\left\|(-\mathscr{A})^{\vartheta} \mathscr{G}(t, 0,0)\right\|_{\mathbb{X}}\right] \\
& \leq \mathcal{M}_{0}\left[L_{\mathscr{G}}\left\|z_{\rho\left(t, z_{t}+y_{t}\right)}+y_{\rho\left(t, z_{t}+y_{t}\right)}\right\|_{\mathscr{B}_{h}}\right. \\
& \left.+\widetilde{L}_{\mathscr{G}}\left\|\int_{0}^{t} e_{1}\left(t, s, z_{\varrho\left(s, z_{s}+y_{s}\right)}+y_{\varrho\left(s, z_{s}+y_{s}\right)}\right) d s\right\|_{\mathbb{X}}+L_{\mathscr{G}}^{*}\right] \\
& \leq \mathcal{M}_{0} L_{\mathscr{G}}\left(\mathscr{D}_{1}^{*} r+c_{n}\right)+\mathcal{M}_{0} \widetilde{L}_{\mathscr{G}} \int_{0}^{t}\left[\| e_{1}\left(t, s, z_{\varrho\left(s, z_{s}+y_{s}\right)}+y_{\varrho\left(s, z_{s}+y_{s}\right)}\right)\right. \\
& \left.-e_{1}(t, s, 0)\left\|_{\mathbb{X}}+\right\| e_{1}(t, s, 0) \|_{\mathbb{X}}\right] d s+\mathcal{M}_{0} L_{\mathscr{G}}^{*} \\
& \leq \mathcal{M}_{0} L_{\mathscr{G}}\left(\mathscr{D}_{1}^{*} r+c_{n}\right)+\mathcal{M}_{0} L_{\mathscr{G}}^{*}+\mathcal{M}_{0} \widetilde{L}_{\mathscr{G}} T\left[L_{e_{1}}\left\|z_{\rho\left(t, z_{t}+y_{t}\right)}+y_{\rho\left(t, z_{t}+y_{t}\right)}\right\|_{\mathscr{B}_{h}}\right. \\
& \left.+L_{e_{1}}^{*}\right] \\
& \leq \mathcal{M}_{0} L_{\mathscr{G}}\left(\mathscr{D}_{1}^{*} r+c_{n}\right)+\mathcal{M}_{0} L_{\mathscr{G}}^{*}+\mathcal{M}_{0} \widetilde{L}_{\mathscr{G}} T L_{e_{1}}\left(\mathscr{D}_{1}^{*} r+c_{n}\right)+\mathcal{M}_{0} \widetilde{L}_{\mathscr{G}} T L_{e_{1}}^{*}, \\
& \| \mathscr{G}\left(t, z_{\varrho\left(t, z_{t}+y_{t}\right)}+y_{\varrho\left(t, z_{t}+y_{t}\right)}, \int_{0}^{t} e_{1}\left(t, s, z_{\varrho\left(s, z_{s}+y_{s}\right)}+y_{\varrho\left(s, z_{s}+y_{s}\right)}\right) d s\right) \\
& -\mathscr{G}\left(t, \bar{z}_{\varrho\left(t, z_{t}+y_{t}\right)}+y_{\varrho\left(t, z_{t}+y_{t}\right)}, \int_{0}^{t} e_{1}\left(t, s, \bar{z}_{\varrho\left(s, z_{s}+y_{s}\right)}+y_{\varrho\left(s, z_{s}+y_{s}\right)}\right) d s\right) \|_{\mathbb{X}}
\end{aligned}
$$

and 
EXACT CONTROLLABILITY OF FRACTIONAL NEUTRAL INTEGRO-DIFFERENTIAL SYSTEMS WITH STATE-DEPENDENT DELAY IN BANACH SPACES

$$
\begin{aligned}
\leq & \left\|(-\mathscr{A})^{-\vartheta}\right\| \|(-\mathscr{A})^{\vartheta} \mathscr{G}\left(t, z_{\varrho\left(t, z_{t}+y_{t}\right)}+y_{\varrho\left(t, z_{t}+y_{t}\right)},\right. \\
& \left.\int_{0}^{t} e_{1}\left(t, s, z_{\varrho\left(s, z_{s}+y_{s}\right)}+y_{\varrho\left(s, z_{s}+y_{s}\right)}\right) d s\right)-(-\mathscr{A})^{\vartheta} \mathscr{G}\left(t, \bar{z}_{\varrho\left(t, z_{t}+y_{t}\right)}+y_{\varrho\left(t, z_{t}+y_{t}\right)},\right. \\
& \left.\int_{0}^{t} e_{1}\left(t, s, \bar{z}_{\varrho\left(s, z_{s}+y_{s}\right)}+y_{\varrho\left(s, z_{s}+y_{s}\right)}\right) d s\right) \|_{\mathbb{X}} \\
\leq & \mathcal{M}_{0}\left[L_{\mathscr{G}}\left\|z_{\varrho\left(t, z_{t}+y_{t}\right)}-\bar{z}_{\varrho\left(t, z_{t}+y_{t}\right)}\right\|_{\mathscr{B}_{h}}+\widetilde{L}_{\mathscr{G}} T L_{e_{1}}\left\|z_{\varrho\left(t, z_{t}+y_{t}\right)}-\bar{z}_{\varrho\left(t, z_{t}+y_{t}\right)}\right\|_{\mathscr{B}_{h}}\right] \\
\leq & \mathcal{M}_{0} \mathscr{D}_{1}^{*}\left[L_{\mathscr{G}}+\widetilde{L}_{\mathscr{G}} T L_{e_{1}}\right]\|z-\bar{z}\|_{\mathscr{B}_{T}^{0}},
\end{aligned}
$$

since

$$
\begin{aligned}
& \left\|z_{\varrho\left(s, z_{s}+y_{s}\right)}-\bar{z}_{\varrho\left(s, z_{s}+y_{s}\right)}\right\| \mathscr{B}_{h} \\
& \leq \mathscr{D}_{1}^{*}|z(s)|+\left(\mathscr{D}_{2}^{*}+J^{\varsigma}\right)\left\|z_{0}\right\|_{\mathscr{B}_{h}}-\mathscr{D}_{1}^{*}|\bar{z}(s)|-\left(\mathscr{D}_{2}^{*}+J^{\varsigma}\right)\left\|\bar{z}_{0}\right\|_{\mathscr{B}_{h}} \\
& \leq \mathscr{D}_{1}^{*}|z(s)-\bar{z}(s)|_{\mathbb{X}} \\
& \leq \mathscr{D}_{1}^{*}\|z-\bar{z}\|_{\mathscr{B}_{T}^{0} .}
\end{aligned}
$$

(iv)

$$
\begin{aligned}
& \| \int_{0}^{t} \mathscr{A}_{\alpha}(t-s) \mathscr{G}\left(s, z_{\varrho\left(s, z_{s}+y_{s}\right)}+y_{\varrho\left(s, z_{s}+y_{s}\right)}\right. \\
& \left.\quad \int_{0}^{s} e_{1}\left(s, \tau, z_{\varrho\left(\tau, z_{\tau}+y_{\tau}\right)}+y_{\varrho\left(\tau, z_{\tau}+y_{\tau}\right)}\right) d \tau\right) d s \|_{\mathbb{X}} \\
& \leq \int_{0}^{t}\left\|(-\mathscr{A})^{1-\vartheta} \mathcal{S}_{\alpha}(t-s)\right\|_{\mathbb{X}}\left[\|(-\mathscr{A})^{\vartheta} \mathscr{G}\left(s, z_{\varrho\left(s, z_{s}+y_{s}\right)}+y_{\varrho\left(s, z_{s}+y_{s}\right)},\right.\right. \\
& \left.\quad \int_{0}^{s} e_{1}\left(s, \tau, z_{\varrho\left(\tau, z_{\tau}+y_{\tau}\right)}+y_{\varrho\left(\tau, z_{\tau}+y_{\tau}\right)}\right) d \tau\right)-(-\mathscr{A})^{\vartheta} \mathscr{G}(s, 0,0) \|_{\mathbb{X}} \\
& \left.\quad+\left\|(-\mathscr{A})^{\vartheta} \mathscr{G}(s, 0,0)\right\|_{\mathbb{X}}\right] d s \\
& \leq \int_{0}^{t} \mathcal{M}(t-s)^{\alpha \vartheta-1}\left[L \mathscr{G}\left\|z_{\varrho\left(s, z_{s}+y_{s}\right)}+y_{\varrho\left(s, z_{s}+y_{s}\right)}\right\|_{\mathscr{B}_{h}}\right. \\
& \quad+\widetilde{L}_{\mathscr{G}} \int_{0}^{s}\left[\left\|e_{1}\left(s, \tau, z_{\varrho\left(\tau, z_{\tau}+y_{\tau}\right)}+y_{\varrho\left(\tau, z_{\tau}+y_{\tau}\right)}\right)-e_{1}(s, \tau, 0)\right\|_{\mathbb{X}}\right. \\
& \left.\left.\quad+\left\|e_{1}(s, \tau, 0)\right\|_{\mathbb{X}}\right] d \tau+L_{\mathscr{G}}^{*}\right] d s
\end{aligned}
$$


EXACT CONTROLLABILITY OF FRACTIONAL NEUTRAL INTEGRO-DIFFERENTIAL SYSTEMS WITH STATE-DEPENDENT DELAY IN BANACH SPACES

$$
\begin{aligned}
& \leq \frac{\mathcal{M} T^{\alpha \vartheta}}{\alpha \vartheta}\left[L_{\mathscr{G}}\left(\mathscr{D}_{1}^{*} r+c_{n}\right)+\widetilde{L}_{\mathscr{G}} T L_{e_{1}}\left(\mathscr{D}_{1}^{*} r+c_{n}\right)+\widetilde{L}_{\mathscr{G}} T L_{e_{1}}^{*}+L_{\mathscr{G}}^{*}\right] \\
& \leq \frac{\mathcal{M} T^{\alpha \vartheta}}{\alpha \vartheta}\left(\mathscr{D}_{1}^{*} r+c_{n}\right)\left\{L_{\mathscr{G}}+\widetilde{L}_{\mathscr{G}} T L_{e_{1}}\right\}+\frac{\mathcal{M} T^{\alpha \vartheta}}{\alpha \vartheta} \widetilde{L}_{\mathscr{G}} T L_{e_{1}}^{*}+\frac{\mathcal{M} T^{\alpha \vartheta}}{\alpha \vartheta} L_{\mathscr{G}}^{*},
\end{aligned}
$$

and

$$
\begin{aligned}
& \| \int_{0}^{t} \mathscr{A} \mathcal{S}_{\alpha}(t-s) \mathscr{G}\left(s, z_{\varrho\left(s, z_{s}+y_{s}\right)}+y_{\varrho\left(s, z_{s}+y_{s}\right)},\right. \\
& \left.\int_{0}^{s} e_{1}\left(s, \tau, z_{\varrho\left(\tau, z_{\tau}+y_{\tau}\right)}+y_{\varrho\left(\tau, z_{\tau}+y_{\tau}\right)}\right) d \tau\right) d s \\
& -\int_{0}^{t} \mathscr{A} \mathcal{S}_{\alpha}(t-s) \mathscr{G}\left(s, \bar{z}_{\varrho\left(s, z_{s}+y_{s}\right)}+y_{\varrho\left(s, z_{s}+y_{s}\right)},\right. \\
& \left.\int_{0}^{s} e_{1}\left(s, \tau, \bar{z}_{\varrho\left(\tau, z_{\tau}+y_{\tau}\right)}+y_{\varrho\left(\tau, z_{\tau}+y_{\tau}\right)}\right) d \tau\right) d s \|_{\mathbb{X}} \\
& \leq \int_{0}^{t}\left\|(-\mathscr{A})^{1-\vartheta} \mathcal{S}_{\alpha}(t-s)\right\|_{\mathbb{X}}\left[\|(-\mathscr{A})^{\vartheta} \mathscr{G}\left(s, z_{\varrho\left(s, z_{s}+y_{s}\right)}+y_{\varrho\left(s, z_{s}+y_{s}\right)},\right.\right. \\
& \left.\int_{0}^{s} e_{1}\left(s, \tau, z_{\varrho\left(\tau, z_{\tau}+y_{\tau}\right)}+y_{\varrho\left(\tau, z_{\tau}+y_{\tau}\right)}\right) d \tau\right) \\
& -(-\mathscr{A})^{\vartheta} \mathscr{G}\left(s, \bar{z}_{\varrho\left(s, z_{s}+y_{s}\right)}+y_{\varrho\left(s, z_{s}+y_{s}\right)},\right. \\
& \left.\left.\int_{0}^{s} e_{1}\left(s, \tau, \bar{z}_{\varrho\left(\tau, z_{\tau}+y_{\tau}\right)}+y_{\varrho\left(\tau, z_{\tau}+y_{\tau}\right)}\right) d \tau\right) \|_{\mathbb{X}}\right] d s \\
& \leq \int_{0}^{t} \mathcal{M}(t-s)^{\alpha \vartheta-1}\left[L \mathscr{G}\left\|z_{\varrho\left(s, z_{s}+y_{s}\right)}-\bar{z}_{\varrho\left(s, z_{s}+y_{s}\right)}\right\|_{\mathscr{B}_{h}}\right. \\
& \left.+\widetilde{L}_{\mathscr{G}} T L_{e_{1}}\left\|z_{\varrho\left(s, z_{s}+y_{s}\right)}-\bar{z}_{\varrho\left(s, z_{s}+y_{s}\right)}\right\|_{\mathscr{B}_{h}}\right] d s \\
& \leq \frac{\mathcal{M} T^{\alpha \vartheta}}{\alpha \vartheta} \mathscr{D}_{1}^{*}\left[L_{\mathscr{G}}+\widetilde{L}_{\mathscr{G}} T L_{e_{1}}\right]\|z-\bar{z}\|_{\mathscr{B}_{T}^{0}} .
\end{aligned}
$$

(v)

$$
\begin{gathered}
\| \int_{0}^{t} \int_{0}^{s} \mathscr{B}(s-\tau) \mathcal{S}_{\alpha}(t-s) \mathscr{G}\left(\tau, z_{\varrho\left(\tau, z_{\tau}+y_{\tau}\right)}+y_{\varrho\left(\tau, z_{\tau}+y_{\tau}\right)},\right. \\
\left.\quad \int_{0}^{\tau} e_{1}\left(\tau, \xi, z_{\varrho\left(\xi, z_{\xi}+y_{\xi}\right)}+y_{\varrho\left(\xi, z_{\xi}+y_{\xi}\right)}\right) d \xi\right) d \tau d s \|_{\mathbb{X}}
\end{gathered}
$$


EXACT CONTROLLABILITY OF FRACTIONAL NEUTRAL INTEGRO-DIFFERENTIAL SYSTEMS WITH STATE-DEPENDENT DELAY IN BANACH SPACES

$$
\begin{aligned}
\leq & \int_{0}^{t} \int_{0}^{s} \mu(s-\tau) \mathcal{M}(t-s)^{\alpha \vartheta-1}\left[\|(-\mathscr{A})^{\vartheta} \mathscr{G}\left(\tau, z_{\varrho\left(\tau, z_{\tau}+y_{\tau}\right)}+y_{\varrho\left(\tau, z_{\tau}+y_{\tau}\right)},\right.\right. \\
& \left.\int_{0}^{\tau} e_{1}\left(\tau, \xi, z_{\varrho\left(\xi, z_{\xi}+y_{\xi}\right)}+y_{\varrho\left(\xi, z_{\xi}+y_{\xi}\right)}\right) d \xi\right)-\left\|(-\mathscr{A})^{\vartheta} \mathscr{G}(\tau, 0,0)\right\|_{\mathbb{X}} \\
& \left.+\left\|(-\mathscr{A})^{\vartheta} \mathscr{G}(\tau, 0,0)\right\|_{\mathbb{X}}\right] d \tau d s \\
\leq & \left(\frac{\mathcal{M} T^{\alpha \vartheta}}{\alpha \vartheta} \int_{0}^{T} \mu(\tau) d \tau\right)\left[L \mathscr{G}\left\|z_{\varrho\left(\tau, z_{\tau}+y_{\tau}\right)}+y_{\varrho}\left(\tau, z_{\tau}+y_{\tau}\right)\right\|_{\mathscr{B}_{h}}\right. \\
& +\widetilde{L}_{\mathscr{G}} \int_{0}^{t}\left[\left\|e_{1}\left(t, s, z_{\varrho\left(s, z_{s}+y_{s}\right)}+y_{\varrho\left(s, z_{s}+y_{s}\right)}\right)-e_{1}(t, s, 0)\right\|_{\mathbb{X}}\right. \\
& \left.\left.+\left\|e_{1}(t, s, 0)\right\|_{\mathbb{X}}\right] d s+L_{\mathscr{G}}^{*}\right] \\
\leq & \left(\frac{\mathcal{M} T^{\alpha \vartheta}}{\alpha \vartheta} \int_{0}^{T} \mu(\tau) d \tau\right)\left[\left(\mathscr{D}_{1}^{*} r+c_{n}\right)\left\{L_{\mathscr{G}}+\widetilde{L}_{\mathscr{G}} T L_{e_{1}}\right\}+\widetilde{L}_{\mathscr{G}} T L_{e_{1}}^{*}+L_{\mathscr{G}}^{*}\right],
\end{aligned}
$$

and

$$
\begin{aligned}
\| & \int_{0}^{t} \int_{0}^{s} \mathscr{B}(s-\tau) \mathcal{S}_{\alpha}(t-s) \mathscr{G}\left(\tau, z_{\varrho\left(\tau, z_{\tau}+y_{\tau}\right)}+y_{\varrho\left(\tau, z_{\tau}+y_{\tau}\right)}\right. \\
& \left.\int_{0}^{\tau} e_{1}\left(\tau, \xi, z_{\varrho\left(\xi, z_{\xi}+y_{\xi}\right)}+y_{\varrho\left(\xi, z_{\xi}+y_{\xi}\right)}\right) d \xi\right) d \tau d s \\
- & \int_{0}^{t} \int_{0}^{s} \mathscr{B}(s-\tau) \mathcal{S}_{\alpha}(t-s) \mathscr{G}\left(\tau, \bar{z}_{\varrho\left(\tau, z_{\tau}+y_{\tau}\right)}+y_{\varrho\left(\tau, z_{\tau}+y_{\tau}\right)},\right. \\
& \left.\int_{0}^{\tau} e_{1}\left(\tau, \xi, \bar{z}_{\varrho\left(\xi, z_{\xi}+y_{\xi}\right)}+y_{\varrho\left(\xi, z_{\xi}+y_{\xi}\right)}\right) d \xi\right) d \tau d s \|_{\mathbb{X}} \\
\leq & \int_{0}^{t} \int_{0}^{s} \mu(s-\tau) \mathcal{M}(t-s)^{\alpha \vartheta-1}\left[\|(-\mathscr{A})^{\vartheta} \mathscr{G}\left(\tau, z_{\varrho\left(\tau, z_{\tau}+y_{\tau}\right)}+y_{\varrho\left(\tau, z_{\tau}+y_{\tau}\right)},\right.\right. \\
& \left.\int_{0}^{\tau} e_{1}\left(\tau, \xi, z_{\varrho\left(\xi, z_{\xi}+y_{\xi}\right)}+y_{\varrho\left(\xi, z_{\xi}+y_{\xi}\right)}\right) d \xi\right) \\
& -(-\mathscr{A})^{\vartheta} \mathscr{G}\left(\tau, \bar{z}_{\varrho\left(\tau, z_{\tau}+y_{\tau}\right)}+y_{\varrho\left(\tau, z_{\tau}+y_{\tau}\right)},\right. \\
& \left.\left.\int_{0}^{\tau} e_{1}\left(\tau, \xi, \bar{z}_{\varrho\left(\xi, z_{\xi}+y_{\xi}\right)}+y_{\varrho\left(\xi, z_{\xi}+y_{\xi}\right)}\right) d \xi\right) \|_{\mathbb{X}}\right] d \tau d s
\end{aligned}
$$


EXACT CONTROLLABILITY OF FRACTIONAL NEUTRAL INTEGRO-DIFFERENTIAL SYSTEMS WITH STATE-DEPENDENT DELAY IN BANACH SPACES

$$
\begin{aligned}
\leq & \left(\frac{\mathcal{M} T^{\alpha \vartheta}}{\alpha \vartheta} \int_{0}^{T} \mu(\tau) d \tau\right)\left[L_{\mathscr{G}}\left\|z_{\varrho\left(t, z_{t}+y_{t}\right)}-\bar{z}_{\varrho\left(t, z_{t}+y_{t}\right)}\right\|_{\mathscr{B}_{h}}\right. \\
& \left.+\widetilde{L}_{\mathscr{G}} T L_{e_{1}}\left\|z_{\varrho\left(t, z_{t}+y_{t}\right)}-\bar{z}_{\varrho\left(t, z_{t}+y_{t}\right)}\right\|_{\mathscr{B}_{h}}\right] \\
\leq & \left(\frac{\mathcal{M} T^{\alpha \vartheta}}{\alpha \vartheta} \int_{0}^{T} \mu(\tau) d \tau\right) \mathscr{D}_{1}^{*}\left[L \mathscr{G}+\widetilde{L} \mathscr{G} T L_{e_{1}}\right]\|z-\bar{z}\|_{\mathscr{B}_{T}^{0}} .
\end{aligned}
$$

(vi)

$$
\begin{aligned}
& \| \int_{0}^{t} \mathcal{S}_{\alpha}(t-s) \mathscr{F}\left(s, z_{\varrho\left(s, z_{s}+y_{s}\right)}+y_{\varrho\left(s, z_{s}+y_{s}\right)},\right. \\
& \left.\quad \int_{0}^{s} e_{2}\left(s, \tau, z_{\varrho\left(\tau, z_{\tau}+y_{\tau}\right)}+y_{\varrho\left(\tau, z_{\tau}+y_{\tau}\right)}\right) d \tau\right) d s \|_{\mathbb{X}} \\
& \leq \int_{0}^{t}\left\|\mathcal{S}_{\alpha}(t-s)\right\|_{\mathscr{L}(\mathbb{X})}\left[\| \mathscr{F}\left(s, z_{\varrho\left(s, z_{s}+y_{s}\right)}+y_{\varrho\left(s, z_{s}+y_{s}\right)},\right.\right. \\
& \left.\quad \int_{0}^{s} e_{2}\left(s, \tau, z_{\varrho\left(\tau, z_{\tau}+y_{\tau}\right)}+y_{\varrho\left(\tau, z_{\tau}+y_{\tau}\right)}\right) d \tau\right) \\
& \left.\quad-\mathscr{F}(s, 0,0)\left\|_{\mathbb{X}}+\right\| \mathscr{F}(s, 0,0) \|_{\mathbb{X}}\right] d s \\
& \leq \mathcal{M} \int_{0}^{t}\left[L \mathscr{F} \| z_{\varrho\left(s, z_{s}+y_{s}\right)}+y_{\varrho\left(s, z_{s}+y_{s}\right)}\right) \mathscr{B}_{h} \\
& \quad+\widetilde{L}_{\mathscr{F}} \int_{0}^{s}\left[\left\|e_{2}\left(s, \tau, z_{\varrho\left(\tau, z_{\tau}+y_{\tau}\right)}+y_{\varrho\left(\tau, z_{\tau}+y_{\tau}\right)}\right)-e_{2}(s, \tau, 0)\right\|_{\mathbb{X}}\right. \\
& \left.\left.\quad+\left\|e_{2}(s, \tau, 0)\right\|_{\mathbb{X}}\right] d \tau+L_{\mathscr{F}}^{*}\right] d s \\
& \leq \mathcal{M} T\left[\left(\mathscr{D}_{1}^{*} r+c_{n}\right)\left\{L \mathscr{F}+\widetilde{L}_{\mathscr{F}} T L_{e_{2}}\right\}+\widetilde{L}_{\mathscr{F}} T L_{e_{2}}+L_{\mathscr{F}}^{*}\right]
\end{aligned}
$$

and

$$
\begin{gathered}
\| \int_{0}^{t} \mathcal{S}_{\alpha}(t-s) \mathscr{F}\left(s, z_{\varrho\left(s, z_{s}+y_{s}\right)}+y_{\varrho\left(s, z_{s}+y_{s}\right)}\right. \\
\left.\int_{0}^{s} e_{2}\left(s, \tau, z_{\varrho\left(\tau, z_{\tau}+y_{\tau}\right)}+y_{\varrho\left(\tau, z_{\tau}+y_{\tau}\right)}\right) d \tau\right) d s
\end{gathered}
$$


EXACT CONTROLLABILITY OF FRACTIONAL NEUTRAL INTEGRO-DIFFERENTIAL SYSTEMS WITH STATE-DEPENDENT DELAY IN BANACH SPACES

$$
\begin{aligned}
& -\int_{0}^{t} \mathcal{S}_{\alpha}(t-s) \mathscr{F}\left(s, \bar{z}_{\varrho\left(s, z_{s}+y_{s}\right)}+y_{\varrho\left(s, z_{s}+y_{s}\right)},\right. \\
& \left.\int_{0}^{s} e_{2}\left(s, \tau, \bar{z}_{\varrho\left(\tau, z_{\tau}+y_{\tau}\right)}+y_{\varrho\left(\tau, z_{\tau}+y_{\tau}\right)}\right) d \tau\right) d s \|_{\mathbb{X}} \\
& \leq \int_{0}^{t}\left\|\mathcal{S}_{\alpha}(t-s)\right\|_{\mathscr{L}(\mathbb{X})}\left[L_{\mathscr{F}}\left\|z_{\varrho\left(s, z_{s}+y_{s}\right)}-\bar{z}_{\varrho\left(s, z_{s}+y_{s}\right)}\right\|_{\mathscr{B}_{h}}\right. \\
& \left.+\widetilde{L}_{\mathscr{F}} T L_{e_{2}}\left\|z_{\varrho\left(s, z_{s}+y_{s}\right)}-\bar{z}_{\varrho\left(s, z_{s}+y_{s}\right)}\right\|_{\mathscr{B}_{h}}\right] d s \\
& \leq \mathcal{M} T \mathscr{D}_{1}^{*}\left\{L_{\mathscr{F}}+\widetilde{L}_{\mathscr{F}} T L_{e_{2}}\right\}\|z-\bar{z}\|_{\mathscr{B}_{T}^{0}} .
\end{aligned}
$$

(vii)

$$
\begin{aligned}
& \left\|\mathrm{e} u_{z+y}(s)\right\| \leq\left(\frac{1}{\gamma} \mathcal{M} \mathcal{M}_{\mathcal{C}}^{2}\right)\left[\left\|x_{T}\right\|+\mathcal{M N}_{0} L \mathscr{G}\|\varsigma\|_{\mathscr{B}_{h}}+\mathcal{M N}_{0} L_{\mathscr{G}}^{*}\right. \\
& +\left(L_{\mathscr{G}}^{*}+\widetilde{L}_{\mathscr{G}} T L_{e_{1}}^{*}\right)\left\{\mathcal{M}_{0}+\frac{\mathcal{M} T^{\alpha \vartheta}}{\alpha \vartheta}\left(1+\int_{0}^{T} \mu(\tau) d \tau\right)\right\} \\
& +\mathcal{M} T\left(L_{\mathscr{F}}^{*}+\widetilde{L}_{\mathscr{F}} T L_{e_{2}}^{*}\right)+\left(\mathscr{D}_{1}^{*} r+c_{n}\right)\left[\mathcal{M} T\left(L_{\mathscr{F}}+\widetilde{L}_{\mathscr{F}} T L_{e_{2}}\right)\right. \\
& \left.\left.+\left\{\mathcal{M}_{0}+\frac{\mathcal{M} T^{\alpha \vartheta}}{\alpha \vartheta}\left(1+\int_{0}^{T} \mu(\tau) d \tau\right)\right\}\left(L_{\mathscr{G}}+\widetilde{L}_{\mathscr{G}} T L_{e_{1}}\right)\right]\right]
\end{aligned}
$$

and

$$
\begin{aligned}
& \left\|\mathcal{C} u_{z+y}(s)-\mathcal{C} u_{\bar{z}+y}(s)\right\| \\
& \leq\left(\frac{1}{\gamma} \mathcal{M} \mathcal{M}_{\mathcal{C}}^{2}\right) \mathscr{D}_{1}^{*}\left[\mathcal{M T} T\left(L_{\mathscr{F}}+\widetilde{L}_{\mathscr{F}} T L_{e_{2}}\right)\right. \\
& \left.\quad+\left\{\mathcal{M}_{0}+\frac{\mathcal{M} T^{\alpha \vartheta}}{\alpha \vartheta}\left(1+\int_{0}^{T} \mu(\tau) d \tau\right)\right\}\left(L_{\mathscr{G}}+\widetilde{L}_{\mathscr{G}} T L_{e_{1}}\right)\right]\|z-\bar{z}\|_{\mathscr{B}_{T}^{0}} .
\end{aligned}
$$

Therefore, we have

$$
\left\|\int_{0}^{t} \mathcal{S}_{\alpha}(t-s) \mathcal{C} u_{z+y}(s) d s\right\|_{\mathbb{X}} \leq \widetilde{C_{1}}+\widetilde{C_{2}},
$$


where

$$
\begin{aligned}
& \widetilde{C_{1}}=\left(\frac{1}{\gamma} \mathcal{M}^{2} \mathcal{M}_{\mathcal{C}}^{2} T\right)\left\|x_{T}\right\|, \\
& \widetilde{C_{2}}=\left(\frac{1}{\gamma} \mathcal{M}^{2} \mathcal{M}_{\mathcal{C}}^{2} T\right)\left[\mathcal{N M N}_{0} L \mathscr{G}\|\varsigma\|_{\mathscr{B}_{h}}+\mathcal{N M N}_{0} L_{\mathscr{G}}^{*}+\left(L_{\mathscr{G}}^{*}+\widetilde{L}_{\mathscr{G}} T L_{e_{1}}^{*}\right)\left\{\mathcal{M}_{0}\right.\right. \\
& \left.+\frac{\mathcal{M} T^{\alpha \vartheta}}{\alpha \vartheta}\left(1+\int_{0}^{T} \mu(\tau) d \tau\right)\right\}+\mathcal{M} T\left(L_{\mathscr{F}}^{*}+\widetilde{L}_{\mathscr{F}} T L_{e_{2}}^{*}\right) \\
& +\left(\mathscr{D}_{1}^{*} r+c_{n}\right)\left[\mathcal{N} T\left(L_{\mathscr{F}}+\widetilde{L}_{\mathscr{F}} T L_{e_{2}}\right)\right. \\
& \left.\left.+\left\{\mathcal{M}_{0}+\frac{\mathcal{M} T^{\alpha \vartheta}}{\alpha \vartheta}\left(1+\int_{0}^{T} \mu(\tau) d \tau\right)\right\}\left(L_{\mathscr{G}}+\widetilde{L}_{\mathscr{G}} T L_{e_{1}}\right)\right]\right],
\end{aligned}
$$

and

$$
\begin{aligned}
& \left\|\int_{0}^{t} \mathcal{S}_{\alpha}(t-s)\left[\mathcal{C} u_{z+y}(s)-\mathfrak{C} u_{\bar{z}+y}(s)\right] d s\right\|_{\mathbb{X}} \\
& \leq\left(\frac{1}{\gamma} \mathcal{M}^{2} \mathcal{M}_{\mathcal{C}}^{2} T\right) \mathscr{D}_{1}^{*}\left[\mathcal{N} T\left(L_{\mathscr{F}}+\widetilde{L}_{\mathscr{F}} T L_{e_{2}}\right)\right. \\
& \left.\quad+\left\{\mathcal{M}_{0}+\frac{\mathcal{M} T^{\alpha \vartheta}}{\alpha \vartheta}\left(1+\int_{0}^{T} \mu(\tau) d \tau\right)\right\}\left(L_{\mathscr{G}}+\widetilde{L}_{\mathscr{G}} T L_{e_{1}}\right)\right]\|z-\bar{z}\|_{\mathscr{B}_{T}^{0}} .
\end{aligned}
$$

Now, we enter the main proof of this theorem. Initially, we demonstrate that $\bar{\Upsilon}$ maps $B_{r}\left(0, \mathscr{B}_{T}^{0}\right)$ into $B_{r}\left(0, \mathscr{B}_{T}^{0}\right)$. For any $z(\cdot) \in \mathscr{B}_{T}^{0}$, by employing Remark 3.1, we sustain

$$
\begin{aligned}
\|(\bar{\Upsilon} z)(t)\|_{\mathbb{X}} & \leq \widetilde{C_{1}}+\widetilde{C_{3}} \\
& \leq r,
\end{aligned}
$$

where

$$
\begin{aligned}
& \widetilde{C_{3}}=\left(1+\frac{1}{\gamma} \mathcal{M}^{2} \mathcal{M}_{\mathcal{C}}^{2} T\right)\left[\mathcal{M N M}_{0} L_{\mathscr{G}}\left\|_{\varsigma}\right\|_{\mathscr{B}_{h}}+\mathcal{M N M}_{0} L_{\mathscr{G}}^{*}+\left(L_{\mathscr{G}}^{*}+\widetilde{L}_{\mathscr{G}} T L_{e_{1}}^{*}\right)\left\{\mathcal{M}_{0}\right.\right. \\
& \left.+\frac{\mathcal{N} T^{\alpha \vartheta}}{\alpha \vartheta}\left(1+\int_{0}^{T} \mu(\tau) d \tau\right)\right\}+\mathcal{N} T\left(L_{\mathscr{F}}^{*}+\widetilde{L}_{\mathscr{F}} T L_{e_{2}}^{*}\right)
\end{aligned}
$$


EXACT CONTROLLABILITY OF FRACTIONAL NEUTRAL INTEGRO-DIFFERENTIAL SYSTEMS WITH STATE-DEPENDENT DELAY IN BANACH SPACES

$$
\begin{aligned}
& +\left(\mathscr{D}_{1}^{*} r+c_{n}\right)\left[\mathcal{M} T\left(L_{\mathscr{F}}+\widetilde{L}_{\mathscr{F}} T L_{e_{2}}\right)\right. \\
& \left.\left.+\left\{\mathcal{M}_{0}+\frac{\mathcal{N} T^{\alpha \vartheta}}{\alpha \vartheta}\left(1+\int_{0}^{T} \mu(\tau) d \tau\right)\right\}\left(L_{\mathscr{G}}+\widetilde{L}_{\mathscr{G}} T L_{e_{1}}\right)\right]\right] .
\end{aligned}
$$

Therefore, $\bar{\Upsilon}$ maps the ball $B_{r}\left(0, \mathscr{B}_{T}^{0}\right)$ into itself. Finally, we show that $\bar{\Upsilon}$ is a contraction on $B_{r}\left(0, \mathscr{B}_{T}^{0}\right)$. For this, let us consider $z, \bar{z} \in B_{r}\left(0, \mathscr{B}_{T}^{0}\right)$, then from Remark 3.1, we sustain

$$
\begin{aligned}
& \|(\bar{\Upsilon} z)(t)-(\bar{\Upsilon} \bar{z})(t)\|_{\mathbb{X}} \\
& \leq\left(1+\frac{1}{\gamma} \mathcal{M}^{2} \mathcal{M}_{\mathfrak{C}}^{2} T\right) \mathscr{D}_{1}^{*}\left[\mathcal{M} T\left(L_{\mathscr{F}}+\widetilde{L}_{\mathscr{F}} T L_{e_{2}}\right)\right. \\
& \left.\quad+\left\{\mathcal{M}_{0}+\frac{\mathcal{M} T^{\alpha \vartheta}}{\alpha \vartheta}\left(1+\int_{0}^{T} \mu(\tau) d \tau\right)\right\}\left(L_{\mathscr{G}}+\widetilde{L}_{\mathscr{G}} T L_{e_{1}}\right)\right] \\
& \leq \Lambda\|z-\bar{z}\|_{\mathscr{B}_{T}^{0}} .
\end{aligned}
$$

From the assumption (H6) and in the perspective of the contraction mapping principle, we understand that $\bar{\Upsilon}$ includes a unique fixed point $z \in \mathscr{B}_{T}^{0}$. Thus, the model (1.1)-(1.2) is exactly controllable on $\mathscr{I}$. The proof is now completed.

\section{Applications}

To exemplify our theoretical outcomes, we treat the FNIDS with SDD of the model

$$
\begin{aligned}
& D_{t}^{\alpha}\left[u(t, x)+\int_{-\infty}^{t} e^{2(s-t)} \frac{u\left(s-\varrho_{1}(s) \varrho_{2}(\|u(s)\|), x\right)}{49} d s\right. \\
& \left.\quad+\int_{0}^{t} \sin (t-s) \int_{-\infty}^{s} e^{2(\tau-s)} \frac{u\left(\tau-\varrho_{1}(\tau) \varrho_{2}(\|u(\tau)\|), x\right)}{36} d \tau d s\right]=\frac{\partial^{2}}{\partial x^{2}} u(t, x) \\
& \quad+\int_{0}^{t}(t-s)^{\delta} e^{-\bar{\gamma}(t-s)} \frac{\partial^{2}}{\partial x^{2}} u(s, x) d s+\nu(t, x) \\
& \quad+\int_{-\infty}^{t} e^{2(s-t)} \frac{u\left(s-\varrho_{1}(s) \varrho_{2}(\|u(s)\|), x\right)}{9} d s
\end{aligned}
$$


EXACT CONTROLLABILITY OF FRACTIONAL NEUTRAL INTEGRO-DIFFERENTIAL SYSTEMS WITH STATE-DEPENDENT DELAY IN BANACH SPACES

$$
\begin{aligned}
& +\int_{0}^{t} \sin (t-s) \int_{-\infty}^{s} e^{2(\tau-s)} \frac{u\left(\tau-\varrho_{1}(\tau) \varrho_{2}(\|u(\tau)\|), x\right)}{25} d \tau d s, \\
u(t, 0)= & 0=u(t, \pi), \quad t \in[0, T], \\
u(t, x)= & \varsigma(t, x), \quad t \leq 0, \quad x \in[0, \pi],
\end{aligned}
$$

where $D_{t}^{\alpha}$ is Caputo's fractional derivative of order $\alpha \in(1,2), \delta$ and $\bar{\gamma}$ are positive numbers and $\varsigma \in \mathscr{B}_{h}$. We consider $\mathbb{X}=L^{2}[0, \pi]$ having the norm $|\cdot|_{L^{2}}$ and determine the operator $\mathscr{A}: D(\mathscr{A}) \subset \mathbb{X} \rightarrow \mathbb{X}$ by $\mathscr{A} w=w^{\prime \prime}$ with the domain

$D(\mathscr{A})=\left\{w \in \mathbb{X}: w, w^{\prime}\right.$ are absolutely continuous, $\left.w^{\prime \prime} \in \mathbb{X}, w(0)=w(\pi)=0\right\}$.

Then

$$
\mathscr{A} w=\sum_{n=1}^{\infty} n^{2}\left\langle w, w_{n}\right\rangle w_{n}, \quad w \in D(\mathscr{A}),
$$

in which $w_{n}(s)=\sqrt{\frac{2}{\pi}} \sin (n s), n=1,2, \ldots$, . is the orthogonal set of eigenvectors of $\mathscr{A}$. It is long familiar that $\mathscr{A}$ is the infinitesimal generator of an analytic semigroup $(T(t))_{t \geq 0}$ in $\mathbb{X}$ and is provided by

$$
T(t) w=\sum_{n=1}^{\infty} e^{-n^{2} t}\left\langle w, w_{n}\right\rangle w_{n}, \quad \text { for all } \quad w \in \mathbb{X}, \quad \text { and every } t>0 .
$$

Hence (H1) is fulfilled. If we fix $\vartheta=\frac{1}{2}$, then the operator $(-\mathscr{A})^{\frac{1}{2}}$ is given by

$$
(-\mathscr{A})^{\frac{1}{2}} w=\sum_{n=1}^{\infty} n\left\langle w, w_{n}\right\rangle w_{n}, \quad w \in\left(D(-\mathscr{A})^{\frac{1}{2}}\right)
$$

in which $\left(D(-\mathscr{A})^{\frac{1}{2}}\right)=\left\{\omega(\cdot) \in \mathbb{X}: \sum_{n=1}^{\infty} n\left\langle\omega, w_{n}\right\rangle w_{n} \in \mathbb{X}\right\}$ and $\left\|(-\mathscr{A})^{-\frac{1}{2}}\right\|=1$. Therefore, $\mathscr{A}$ is sectorial of type and the properties (P1) hold. We also take into account the operator $\mathscr{B}(t): D(\mathscr{A}) \subseteq \mathbb{X} \rightarrow \mathbb{X}, t \geq 0, \mathscr{B}(t) x=t^{\delta} e^{-\bar{\gamma} t} \mathscr{A} x$ for $x \in D(\mathscr{A})$. In addition, it is simple to see that conditions (P2)-(P3)[for more details, refer[22]] are fulfilled with $b(t)=t^{\delta} e^{-\bar{\gamma} t}$ and $D=C_{0}^{\infty}([0, \pi])$, where $C_{0}^{\infty}([0, \pi])$ is the space of infinitely differentiable functions that vanish at $x=0$ and $x=\pi$. From the Lemma 2.4, it is simple to see that condition (H2) is fulfills.

For the phase space, we choose $h=e^{2 s}, s<0$, then $l=\int_{-\infty}^{0} h(s) d s=\frac{1}{2}<$ $\infty$, for $t \leq 0$ and determine

$$
\|\varsigma\|_{\mathscr{B}_{h}}=\int_{-\infty}^{0} h(s) \sup _{\theta \in[s, 0]}\|\varsigma(\theta)\|_{L^{2}} d s .
$$


Hence, for $(t, \varsigma) \in[0,1] \times \mathscr{B}_{h}$, where $\varsigma(\theta)(x)=\varsigma(\theta, x),(\theta, x) \in(-\infty, 0] \times$ $[0, \pi]$. Set

$$
u(t)(x)=u(t, x), \quad \varrho(t, \varsigma)=\varrho_{1}(t) \varrho_{2}(\|\varsigma(0)\|),
$$

we have

$$
\begin{gathered}
\mathscr{G}(t, \varsigma, \overline{\mathscr{H}} \varsigma)(x)=\int_{-\infty}^{0} e^{2(s)} \frac{\varsigma}{49} d s+(\overline{\mathscr{H}} \varsigma)(x), \\
\mathscr{F}(t, \varsigma, \mathscr{H} \varsigma)(x)=\int_{-\infty}^{0} e^{2(s)} \frac{\varsigma}{9} d s+(\mathscr{H} \varsigma)(x),
\end{gathered}
$$

where

$$
\begin{aligned}
& \left(\overline{\mathscr{H}}_{\varsigma}\right)(x)=\int_{0}^{t} \sin (t-s) \int_{-\infty}^{0} e^{2(\tau)} \frac{\varsigma}{36} d \tau d s, \\
& (\mathscr{H} \varsigma)(x)=\int_{0}^{t} \sin (t-s) \int_{-\infty}^{0} e^{2(\tau)} \frac{\varsigma}{25} d \tau d s .
\end{aligned}
$$

Further, we define the operator $\mathcal{C}: U \rightarrow \mathbb{X}$ by $\mathcal{C} u(t, x)=\nu(t, x), 0<x<\pi, u \in$ $U$, where $\nu:[0,1] \times[0, \pi] \rightarrow[0, \pi]$, then using these configurations, the system (4.1)-(4.3) is usually written in the theoretical form of design (1.1)-(1.2).

To treat this system we assume that $\varrho_{i}:[0, \infty) \rightarrow[0, \infty), i=1,2$ are continuous. Now, we can see that for $t \in[0,1], \varsigma, \bar{\varsigma} \in \mathscr{B}_{h}$, we have

$$
\begin{aligned}
& \left\|(-\mathscr{A})^{\frac{1}{2}} \mathscr{G}(t, \varsigma, \overline{\mathscr{H}} \varsigma)\right\|_{\mathbb{X}} \\
& \leq\left(\int_{0}^{\pi}\left(\int_{-\infty}^{0} e^{2(s)}\left\|\frac{\varsigma}{49}\right\| d s+\int_{0}^{t}\|\sin (t-s)\| \int_{-\infty}^{0} e^{2(\tau)}\left\|\frac{\varsigma}{36}\right\| d \tau d s\right)^{2} d x\right)^{\frac{1}{2}} \\
& \leq\left(\int_{0}^{\pi}\left(\frac{1}{49} \int_{-\infty}^{0} e^{2(s)} \sup \|\varsigma\| d s+\frac{1}{36} \int_{-\infty}^{0} e^{2(s)} \sup \|\varsigma\| d s\right)^{2} d x\right)^{\frac{1}{2}} \\
& \leq \frac{\sqrt{\pi}}{49}\|\varsigma\|_{\mathscr{B}_{h}}+\frac{\sqrt{\pi}}{36}\|\varsigma\|_{\mathscr{B}_{h}} \\
& \leq L \mathscr{G}\left\|_{\mathscr{B}_{h}}+\widetilde{L}_{\mathscr{G}}\right\|_{\mathscr{B}} \|_{\mathscr{B}_{h}},
\end{aligned}
$$

where $L_{\mathscr{G}}+\widetilde{L}_{\mathscr{G}}=\frac{85 \sqrt{\pi}}{1764}$, and

$$
\begin{aligned}
& \left\|(-\mathscr{A})^{\frac{1}{2}} \mathscr{G}(t, \varsigma, \overline{\mathscr{H}} \varsigma)-(-\mathscr{A})^{\frac{1}{2}} \mathscr{G}(t, \bar{\varsigma}, \overline{\mathscr{H}} \bar{\varsigma})\right\|_{\mathbb{X}} \\
& \leq\left(\int _ { 0 } ^ { \pi } \left(\int_{-\infty}^{0} e^{2(s)}\left\|\frac{\varsigma}{49}-\frac{\bar{\varsigma}}{49}\right\| d s\right.\right.
\end{aligned}
$$




$$
\begin{aligned}
& \left.\left.+\int_{0}^{t}\|\sin (t-s)\| \int_{-\infty}^{0} e^{2(\tau)}\left\|\frac{\varsigma}{36}-\frac{\bar{\varsigma}}{36}\right\| d \tau d s\right)^{2} d x\right)^{\frac{1}{2}} \\
\leq & \left(\int_{0}^{\pi}\left(\frac{1}{49} \int_{-\infty}^{0} e^{2(s)} \sup \|\varsigma-\bar{\varsigma}\| d s+\frac{1}{36} \int_{-\infty}^{0} e^{2(s)} \sup \|\varsigma-\bar{\varsigma}\| d s\right)^{2} d x\right)^{\frac{1}{2}} \\
\leq & \frac{\sqrt{\pi}}{49}\|\varsigma-\bar{\varsigma}\|_{\mathscr{B}_{h}}+\frac{\sqrt{\pi}}{36}\|\varsigma-\bar{\varsigma}\|_{\mathscr{B}_{h}} \\
\leq & L \mathscr{G}\|\varsigma-\bar{\varsigma}\|_{\mathscr{B}_{h}}+\widetilde{L} \mathscr{G}\left\|_{\varsigma}-\bar{\varsigma}\right\|_{\mathscr{B}_{h}} .
\end{aligned}
$$

Similarly, we conclude

$$
\begin{aligned}
& \|\mathscr{F}(t, \varsigma, \mathscr{H} \varsigma)\|_{L^{2}} \\
& \leq\left(\int_{0}^{\pi}\left(\int_{-\infty}^{0} e^{2(s)}\left\|\frac{\varsigma}{9}\right\| d s+\int_{0}^{t}\|\sin (t-s)\| \int_{-\infty}^{0} e^{2(\tau)}\left\|\frac{\varsigma}{25}\right\| d \tau d s\right)^{2} d x\right)^{\frac{1}{2}} \\
& \leq\left(\int_{0}^{\pi}\left(\frac{1}{9} \int_{-\infty}^{0} e^{2(s)} \sup \|\varsigma\| d s+\frac{1}{25} \int_{-\infty}^{0} e^{2(s)} \sup \|\varsigma\| d s\right)^{2} d x\right)^{\frac{1}{2}} \\
& \leq \frac{\sqrt{\pi}}{9}\|\varsigma\|_{\mathscr{B}_{h}}+\frac{\sqrt{\pi}}{25}\|\varsigma\|_{\mathscr{B}_{h}} \\
& \leq L \mathscr{F}\left\|_{\mathscr{B}_{h}}+\widetilde{L}_{\mathscr{F}}\right\| \varsigma \|_{\mathscr{B}_{h}},
\end{aligned}
$$

where $L_{\mathscr{F}}+\widetilde{L}_{\mathscr{F}}=\frac{34 \sqrt{\pi}}{225}$, and

$$
\begin{aligned}
\|\mathscr{F}(t, \varsigma, \mathscr{H} \varsigma)-\mathscr{F}(t, \bar{\varsigma}, \mathscr{H} \bar{\varsigma})\|_{L^{2}} \\
\leq\left(\int _ { 0 } ^ { \pi } \left(\int_{-\infty}^{0} e^{2(s)}\left\|\frac{\varsigma}{9}-\frac{\bar{\varsigma}}{9}\right\| d s\right.\right. \\
\left.\left.\quad+\int_{0}^{t}\|\sin (t-s)\| \int_{-\infty}^{0} e^{2(\tau)}\left\|\frac{\varsigma}{25}-\frac{\bar{\varsigma}}{25}\right\| d \tau d s\right)^{2} d x\right)^{\frac{1}{2}} \\
\leq\left(\int_{0}^{\pi}\left(\frac{1}{9} \int_{-\infty}^{0} e^{2(s)} \sup \|\varsigma-\bar{\varsigma}\| d s+\frac{1}{25} \int_{-\infty}^{0} e^{2(s)} \sup \|\varsigma-\bar{\varsigma}\| d s\right)^{2} d x\right)^{\frac{1}{2}} \\
\leq \frac{\sqrt{\pi}}{9}\|\varsigma-\bar{\varsigma}\|_{\mathscr{B}_{h}}+\frac{\sqrt{\pi}}{25}\|\varsigma-\bar{\varsigma}\|_{\mathscr{B}_{h}} \\
\leq L{ }_{\mathscr{F}}\|\varsigma-\bar{\varsigma}\|_{\mathscr{B}_{h}}+\widetilde{L}_{\mathscr{F}}\|\varsigma-\bar{\varsigma}\|_{\mathscr{B}_{h}} .
\end{aligned}
$$

Therefore the conditions (H3) and (H5) are all fulfilled. Furthermore, we assume that $\mathscr{D}_{1}^{*}=\frac{1}{2}, \mathcal{M}_{0}=1, \mathcal{M}=1, \mathcal{M}_{\mathcal{C}}=1, \gamma=1, T=1, \alpha=\frac{3}{2}, L_{e_{1}}=$ 


$$
\begin{aligned}
& 1, L_{e_{2}}=1 \text { and } \int_{0}^{1} \mu(\tau) d \tau=1 \text {. Then } \\
& \left(1+\frac{1}{\gamma} \mathcal{M}^{2} \mathcal{M}_{\mathcal{C}}^{2} T\right) \mathscr{D}_{1}^{*}\left[\mathcal{M} T\left(L_{\mathscr{F}}+\widetilde{L}_{\mathscr{F}} T L_{e_{2}}\right)\right. \\
& \left.\quad+\left\{\mathcal{M}_{0}+\frac{\mathcal{M} T^{\alpha \vartheta}}{\alpha \vartheta}\left(1+\int_{0}^{T} \mu(\tau) d \tau\right)\right\}\left(L_{\mathscr{G}}+\widetilde{L}_{\mathscr{G}} T L_{e_{1}}\right)\right] \approx 0.58098<1 .
\end{aligned}
$$

Thus the condition (H6) holds. Hence by Theorem 3.1, we realize that the system (4.1)-(4.3) has a unique mild solution on $[0,1]$.

\section{References}

[1] D. Baleanu, J. A. T. Machado, A. C. J. Luo, Fractional Dynamics and Control, Springer, New York, USA, 2012.

[2] A. Kilbas, H. Srivastava and J. J. Trujillo, Theory and Applications of Fractional Differential Equations, Elsevier, Amesterdam, 2006.

[3] G. Bonanno, R. Rodriguez-Lopez, S. Tersian, Existence of solutions to boundary value problem for impulsive fractional differential equations, Fractional Calculus and Applied Analysis, 17(3)(2014), 717-744.

[4] R. Rodriguez-Lopez and S. Tersian, Multiple solutions to boundary value problem for impulsive fractional differential equations, Fractional Calculus and Applied Analysis, 17(4)(2014), 1016-1038.

[5] R. P. Agarwal, V. Lupulescu, D. O'Regan and G. Rahman, Fractional calculus and fractional differential equations in nonreflexive Banach spaces, Communications in Nonlinear Science and Numerical Simulation, 20(1)(2015), 59-73.

[6] E. Keshavarz, Y. Ordokhani and M. Razzaghi, Bernoulli wavelet operational matrix of fractional order integration and its applications in solving the fractional order differential equations, Applied Mathematical Modelling, 38(2014), 6038-6051.

[7] Z. Lv and B. Chen, Existence and uniqueness of positive solutions for a fractional switched system, Abstract and Applied Analysis, Volume 2014 (2014), Article ID 828721, 7 pages. 
EXACT CONTROLLABILITY OF FRACTIONAL NEUTRAL INTEGRO-DIFFERENTIAL SYSTEMS WITH STATE-DEPENDENT DELAY IN BANACH SPACES

[8] Y. Wang, L. Liu and Y. Wu, Positive solutions for a class of higher-order singular semipositone fractional differential systems with coupled integral boundary conditions and parameters, Advances in Difference Equations, 2014:268, 1-24.

[9] B. Ahmad, S. K. Ntouyas and A. Alsaed, Existence of solutions for fractional q-integro-difference inclusions with fractional q-integral boundary conditions, Advances in Difference Equations, 2014:257, 1-18.

[10] R. P. Agarwal, B. D. Andrade, On fractional integro-differential equations with state-dependent delay, Comp. Math. App., 62(2011), 1143-1149.

[11] M. Benchohra, F. Berhoun, Impulsive fractional differential equations with state-dependent delay, Commun. Appl. Anal., 14(2)(2010), 213-224.

[12] K. Aissani and M. Benchohra, Fractional integro-differential equations with state-dependent delay, Advances in Dynamical Systems and Applications, 9(1)(2014), 17-30.

[13] J. Dabas and G.R. Gautam, Impulsive neutral fractional integrodifferential equation with state-dependent delay and integral boundary condition, Electronic Journal of Differential Equations, Vol. 2013 (2013), No. 273, 1-13.

[14] S. Suganya, M. Mallika Arjunan, and J. J. Trujillo, Existence results for an impulsive fractional integro-differential equation with state-dependent delay, Applied Mathematics and Computation, 266(2015), 54-69.

[15] J. P. C. Dos Santos, C. Cuevas, B. de Andrade, Existence results for a fractional equations with state-dependent delay, Advances in Difference Equations, (2011), 1-15.

[16] J. P. C. Dos Santos, M. Mallika Arjunan, and C. Cuevas, Existence results for fractional neutral integrodifferential equations with state-dependent delay, Comput. Math. Appl., 62(2011), 1275-1283.

[17] Z. Yan, Approximate controllability of fractional neutral integrodifferential inclusions with state-dependent delay in Hilbert spaces, IMA Journal of Mathematical Control and Information, 30(2013), 443-462.

[18] Z. Yan and X. Jia, Approximate controllability of partial fractional neutral stochastic functional integro-differential inclusions with statedependent delay, Collect. Math., 66(2015), 93-124. 
EXACT CONTROLLABILITY OF FRACTIONAL NEUTRAL INTEGRO-DIFFERENTIAL SYSTEMS WITH STATE-DEPENDENT DELAY IN BANACH SPACES

[19] V. Vijayakumar, C. Ravichandran and R. Murugesu, Approximate controllability for a class of fractional neutral integro-differential inclusions with state-dependent delay, Nonlinear Studies, 20(4)(2013), 513-532.

[20] R. P. Agarwal, J. P. C. Dos Santos, and C. Cuevas, Analytic resolvent operator and existence results for fractional integro-differential equations, J. Abstr. Differ. Equ. Appl., 2(2)(2012), 26-47.

[21] V. Vijayakumar, A. Selvakumar and R. Murugesu, Controllability for a class of fractional neutral integro-differential equations with unbounded delay, Applied Mathematics and Computation, 232(2014), 303-312.

[22] B. D. Andrade, J. P. C. Dos Santos, Existence of solutions for a fractional neutral integro-differential equation with unbounded delay, Electronic Journal of Differential Equations, Vol. 2012 (2012), No. 90, pp. 1-13.

[23] J. P. C. Dos Santos, V. Vijayakumar and R. Murugesu, Existence of mild solutions for nonlocal Cauchy problem for fractional neutral integro-differential equation with unbounded delay, Communications in Mathematical Analysis, X(2011), 1-13.

[24] X. Shu and Q. Wang, The existence and uniqueness of mild solutions for fractional differential equations with nonlocal conditions of order $1<\alpha<2$, Comput. Math. Appl., 64(2012), 2100-2110.

[25] Z. Yan and X. Jia, Impulsive problems for fractional partial neutral functional integro-differential inclusions with infinite delay and analytic resolvent operators, Mediterr. Math., 11(2014), 393-428.

[26] Z. Yan and F. Lu, On approximate controllability of fractional stochastic neutral integro-differential inclusions with infinite delay, Applicable Analysis, (2014), 1235-1258.

[27] L. Kexue, P. Jigen, G. Jinghuai, Controllability of nonlocal fractional differential systems of order $\alpha \in(1,2]$ in Banach spaces, Rep. Math. Phys. , 71(2013), 33-43.

[28] R. Sakthivel, R. Ganesh, Y. Ren, S. Marshal Anthoni, Approximate controllability of nonlinear fractional dynamical systems, Commun. Nonlinear Sci. Numer. Simulat., 18(2013), 3498-3508.

[29] C. Rajivganthi, P. Muthukumar. and B. Ganesh Priya, Approximate controllability of fractional stochastic integro-differential equations with infinite delay of order $1<\alpha<2$, IMA Journal of Mathematical Control and Information, (2015), 1-15, Available On line. 
EXACT CONTROLLABILITY OF FRACTIONAL NEUTRAL INTEGRO-DIFFERENTIAL SYSTEMS WITH STATE-DEPENDENT DELAY IN BANACH SPACES

[30] J. Dabas and A. Chauhan, Existence and uniqueness of mild solution for an impulsive fractional integro-differential equation with infinite delay, Mathematical and Computer Modelling, 57(3-4)(2013), 754-763.

[31] J. Hale and J. Kato, Phase space for retarded equations with infinite delay, Funkcial. Ekvac., 21(1978), 11-41.

[32] Y. Hino, S. Murakami, and T. Naito, Functional Differential Equations with Unbounded Delay, Springer-Verlag, Berlin, 1991.

[33] X. Fu and R. Huang, Existence of solutions for neutral integro-differential equations with state-dependent delay, Appl. Math. Comp., 224(2013), 743-759.

[34] A. Pazy, Semigroups of Linear Operators and Applications to Partial Differential Equations, Springer-Verlag, New York, 1983.

[35] N. I. Mahmudov and A. Denker, On controllability of linear stochastic systems, Int. J. Control, 73(2000), 144-151.

Subramanian Kailasavalli,

Department of Mathematics,

PSNA College of Engineering and Technology,

Dindigul-624622, Tamil Nadu, India.

Email: skvalli2k5@gmail.com

Dumitru Baleanu,

Department of Mathematics and Computer Sciences,

Faculty of Art and Sciences,

Cankaya University, 06530 Ankara,

Turkey and Institute of Space Sciences,

Magurele-Bucharest, Romania.

Email: dumitru@cankaya.edu.tr

Selvaraj Suganya,

Department of Mathematics,

C. B. M. College, Kovaipudur,

Coimbatore - 641 042, Tamil Nadu, India.

Email: selvarajsuganya2014@yahoo.in

Mani Mallika Arjunan,

Department of Mathematics,

C. B. M. College, Kovaipudur,

Coimbatore - 641 042, Tamil Nadu, India.

Email: arjunphd07@yahoo.co.in 